University of Massachusetts Amherst

ScholarWorks@UMass Amherst

Chemical Engineering Faculty Publication Series

Chemical Engineering

2015

\title{
PRISM-Based Theory of Complex Coacervation: Excluded Volume versus Chain Correlation
}

\author{
Sarah L. Perry \\ University of Massachusetts Amherst \\ Charles E. Sing \\ University of Illinois at Urbana-Champaign
}

Follow this and additional works at: https://scholarworks.umass.edu/che_faculty_pubs

Part of the Chemical Engineering Commons

\section{Recommended Citation}

Perry, Sarah L. and Sing, Charles E., "PRISM-Based Theory of Complex Coacervation: Excluded Volume versus Chain Correlation" (2015). Macromolecules. 832.

https://doi.org/10.1021/acs.macromol.5b01027

This Article is brought to you for free and open access by the Chemical Engineering at ScholarWorks@UMass Amherst. It has been accepted for inclusion in Chemical Engineering Faculty Publication Series by an authorized administrator of ScholarWorks@UMass Amherst. For more information, please contact scholarworks@library.umass.edu. 


\title{
PRISM-based Theory of Complex
}

\section{Coacervation: Excluded Volume versus Chain Correlation}

\author{
Sarah L. Perry ${ }^{*, \dagger}$ and Charles E. Sing ${ }^{*, \dagger}$ \\ Department of Chemical Engineering, University of Massachusetts Amherst, Amherst, MA \\ 01003, and Department of Chemical and Biomolecular Engineering, University of Illinois \\ Urbana-Champaign, Urbana, IL 61801
}

E-mail: perrys@engin.umass.edu; cesing@illinois.edu

\begin{abstract}
Aqueous solutions of oppositely-charged polyelectrolytes can undergo liquid-liquid phase separation into materials known as complex coacervates. These coacervates have been a subject of intense experimental and theoretical interest. Efforts to provide a physical description of complex coacervates have led to a number of theories that qualitatively (and sometimes quantitatively) agree with experimental data. However, this agreement often occurs in a degeneracy of models with profoundly different starting assumptions and different levels of sophistication. Theoretical difficulties in these systems are similar to those in most polyelectrolyte systems where charged species are highly correlated. These highly-correlated systems can be described using Liquid State (LS) integral equation theories, which surpass mean field theories by providing information

${ }^{*}$ To whom correspondence should be addressed

${ }^{\dagger}$ University of Massachusetts Amherst

$\ddagger$ University of Illinois Urbana-Champaign
\end{abstract}


on local charge ordering. We extend these ideas to complex coacervate systems using PRISM-type theories, and are able to capture effects not observable in traditional coacervate models, particularly connectivity and excluded volume effects. We can thus bridge two traditional but incommensurate theories meant to describe complex coacervates: the Voorn-Overbeek theory and counterion release. Importantly, we hypothesize that a cancellation of connectivity and excluded volume effects provides an explanation for the ability of Voorn-Overbeek theory to fit experimental data despite its well-known approximations.

\section{Introduction}

Complex coacervates are a long-studied class of materials that are typically formed from more than one oppositely charged species. ${ }^{1-3}$ By definition, the opposite charge on the two species results in a 'complex.' Furthermore, the nature of the charged species must allow for the formation of a 'coacervate', which represents a subset of complexes that undergo a liquid-liquid phase separation rather than forming solid precipitates. ${ }^{1-6}$ Historical and current investigations into complex coacervates have studied the formation of such phases in natural polymers, such as proteins or polysaccharides, which are currently widely used as food additives. ${ }^{7-18}$ Despite the utility of these systems, there has only recently been a resurgence of interest in their molecular behavior, in particular for its promise as a powerful route to self-assembled materials such as micelles, ${ }^{3,19-22}$ block copolymers, ${ }^{23-26}$ and layer-bylayer assembly. ${ }^{27-30}$ This recent activity in the field is concomitant with a desire to emulate the molecular features observed in a number of biological materials, such as underwater adhesives in mussels and the matrix adhesive holding together the dwelling of a sandcastle worm. ${ }^{2,31-33}$ The novelty of these materials is their extreme stability (yet responsiveness) with respect to environmental ionic conditions, even at high salt concentrations, as well as their

reversible assembly behavior. ${ }^{34-38}$ Complex coacervates are used for microencapsulation, drug delivery, ${ }^{3,19}$ biomaterials, ${ }^{39-41}$ and underwater adhesives, ${ }^{2,31,32}$ where their self assembly and 
functionality relies ${ }^{19,23-26}$ on the relationship between charged species. Thus, the molecular features of these charged species is crucial to designing function. Theoretical work should correspondingly relate to these molecular features in a fashion that permits their design.

While complex coacervate materials may be comprised of any number of component types, such as colloids or folded proteins, ${ }^{42-46}$ we will primarily focus on flexible, charged polymers. These systems typically have four non-water components, two oppositely-charged polyions and two oppositely-charged salt ions. ${ }^{1}$ Polymeric complex coacervates demonstrate a rich phenomenology that is a function of the presence of salt as well as the nature of the charged polyions, ${ }^{1,32,34-38,47-50}$ with a vast parameter space that spans the molecular features of all four components. Experimentalists have begun to systematically explore this space, looking at effects such as chain solubility and length, ion identity, and valency. ${ }^{1,32,34-38,47-51}$ Nevertheless, a comprehensive physical picture of these systems remains to be elucidated. These systems are conceptually portrayed in a variety of ways. Physical principles ranging from counterion release ${ }^{44,52-54}$ to electrostatic screening, ${ }^{7-9}$ to two-step models (and permutations thereof) ${ }^{10,36,42}$ are all invoked to some degree of success, however there remains some ambiguity over the physical nature of coacervation.

This ambiguity is compounded by the wide variety of theoretical models purporting to describe complex coacervation. The initial picture, which prevails to most modern studies, was established more than 50 years ago by Voorn and Overbeek. ${ }^{7,8}$ This eponymous theory describes a competition between the mixing entropy and dispersive interactions of the polymers, introduced via a Flory-Huggins term, ${ }^{55}$ and the electrostatic interaction between the oppositely-charged polyions, introduced via a Debye-Hückel term. ${ }^{7,8,56}$ The free energy $F_{V O}$ of a complex coacervate system of volume $V$ at temperature $T$ is given by: ${ }^{7,8}$

$$
\frac{F_{V O}}{k_{B} T}=V\left[\frac{\phi_{ \pm}}{N} \ln \phi_{ \pm}+\left(1-\phi_{ \pm}\right) \ln \left(1-\phi_{ \pm}\right)+\chi \phi_{ \pm}\left(1-\phi_{ \pm}\right)-\frac{2 \sqrt{\pi}}{3} \Gamma^{3 / 2} \phi_{ \pm}^{3 / 2}\right]
$$

where $\phi_{ \pm}$is the combined volume fraction of polyion with length $N$, and $\Gamma=e^{2} /\left(8 \pi \epsilon a k_{B} T\right)$ 
is the parameter describing the magnitude of the electrostatic interaction in the electrolyte solution with dielectric constant $\epsilon$ and salt ion size $a$. The incorporation of a polymer-solvent $\chi$ parameter is a modification of the original theory, ${ }^{9}$ and can be relevant for synthetic polymers that are inherently water-insoluble. Excellent agreement between experiment and theory is possible ${ }^{34}$ and extensions to (for example) adapt Cahn-Hilliard models of interfaces can describe interfacial surface tension effects. ${ }^{57}$ However this theory carries with it the approximations inherent to the linearized, mean field Debye-Hückel theory. ${ }^{56}$

Non-mean field behaviors are expected and experimentally realized in most real polyelectrolyte systems, ${ }^{58-60}$ especially at the high salt concentrations prevalent in coacervate systems. ${ }^{61,62}$ Extensions to rectify this have been proposed. ${ }^{62,63}$ The mean-field nature of the Voorn-Overbeek model has been relaxed in both theory ${ }^{64}$ as well as elaborate numerical theory by Fredrickson et al. ${ }^{66-68}$ In the latter, fluctuations are incorporated into both the electrostatic as well as the polymer degrees of freedom using complex Langevin methods. ${ }^{66,67}$ In principle, such methods should be able to fully describe coacervate systems as they are not restrained by the "mean field" assumptions that the Voorn-Overbeek theory carries with it, and the Hamiltonian in these systems is essentially complete. ${ }^{66,67}$ In practice, however, these theories require a finite-sized grid for numerical simulation, setting a "resolution" below which it is difficult to articulate correlations.

Despite these significant advances, alternative models have been developed that rely on different, often more phenomenological ideas. For example, simple arguments based on "counterion release" have been successfully implemented both in theory and experiment. ${ }^{52-54}$ These rely on the presence of Manning condensation, ${ }^{69}$ which provides a local abundance of salt charges near isolated polyions. Upon complexation between two polyions, these "bound" or "condensed" counterions now are free to translate through the entire system. ${ }^{15,52,70}$ Such arguments are fundamentally different from Voorn-Overbeek related models. Namely, they require the presence of highly-connected charges (in the form of the polyions), which are essentially absent in the original Voorn-Overbeek theory. ${ }^{7,8}$ 
While fluctuating field methods are a significant improvement, ${ }^{66,67}$ and Random Phase Approximation (RPA)-based theories have provided promising alternatives that surpass Voorn-Overbeek to include correlation and excluded volume effects, ${ }^{71,72}$ there remains an urgent need for new theoretical insight. Most importantly, there is a need to understand how these two disparate theoretical approaches relate to each other. The Voorn-Overbeek theory has shown excellent agreement with data, ${ }^{34,73}$ despite its well-known theoretical deficiencies and disregard for the features that are the driving concepts in counterion-release arguments. ${ }^{15}$ There is a need to understand this paradoxical state of the theory.

An alternative to Vorn-Overbeek or field theories is desired in order to clarify the role of molecular details such as connectivity. One promising route is to use liquid state theory. Liquid state (LS) theories are a powerful class of theories that are capable of incorporating the features of highly-correlated systems, which are challenging or intractable with mean-field approaches. ${ }^{74}$ They are the standard theoretical approach for liquids, and represent an alternative to prior approaches to describe coacervates due to their ability to articulate correlations in systems such as charged electrolyte and polyelectrolyte systems. ${ }^{74-78}$ In particular, LS can often reproduce pair correlations at a quantitative level, typically evidenced by comparing to full Molecular Dynamics simulations. ${ }^{74,75,79}$ Even in situations where quantitative matching fails, qualitative features are typically observed. ${ }^{74,79}$ The calculation of these pair correlations provides the information needed to determine macroscopic thermodynamic parameters such as energy, pressure, or chemical potential. ${ }^{74}$

For polymeric systems, connectivity can be included via modifications to LS theory, through an extension known as the Polymer Reference Interaction Site Model (PRISM). ${ }^{80}$ This model introduces connected correlations, with each monomer along the chain 'observing' neighbors as bonded in the same fashion (i.e. a monomer far from a chain end will on average experience the same 
environment). ${ }^{77,80}$ For coacervate systems, this uniquely enables articulation of local correlations with respect to molecular features such as connectivity while providing the information to calculate macroscopic thermodynamics necessary to understand phase behavior. There are disadvantages to PRISM, in particular it typically requires a homogeneous, isotropic system and is often numerically challenging. ${ }^{74}$ Furthermore, it relies on non-exact 'closure relations' whose opaque approximations are difficult to conceptualize and improve upon. ${ }^{74} \mathrm{PRISM}$ is nevertheless able to consider local connectivity and structure that is difficult or intractable in prior methods, and for complex coacervates is able to provide insights not apparent in field theory-based approaches.

In this paper, we develop a PRISM-based theory that provides an initial look at molecularlevel charge correlations in the context of complex coacervate phase behavior. We will probe this model and demonstrate how two important and competing effects (excluded volume and chain connectivity), which are neglected in the Voorn-Overbeek model, ${ }^{7,8}$ cancel out such that the Voorn-Overbeek model seemingly produces excellent fits in regimes far outside its theoretical applicability. This allows us to develop new predictions for measurable quantities such as salt partitioning and chain connectivity effects on coacervate phase behavior. We show how molecular features of either the polyions or the salt ions may be harnessed to promote or prevent the formation of complex coacervates.

\section{Theory}

Recent advances in the theoretical description of polyelectrolyte materials in gels, ${ }^{62}$ blends, ${ }^{63,81}$ and block copolymers ${ }^{81,82}$ have succeeded in incorporating charge correlations into thermodynamic models in a multiscale fashion. Importantly, these new theoretical tools enable the articulation of molecular structure at the level of the so-called "primitive model" that considers charged components as spherical beads. While this ostensibly neglects some atomistic-level 
features, the local organization of even these simplified charged species has been demonstrated to have profound effects on the thermodynamics of materials. ${ }^{58-60,62,63,81,82}$ Many of these systems maintain charge ordering due to the high strength of Coulombic attractions between opposite charges; in polymer blends and block copolymers this is due to an extremely low relative dielectric constant $\epsilon_{r}<10,{ }^{63,81,82}$ and in single-polyelectrolyte solutions this is due to highly-valent salts. ${ }^{59,60,62}$ For complex coacervates, we are often under conditions of high dielectric constant $\left(\epsilon_{r}=80.1\right)$ and low valency $z \sim \pm 1$. Despite being in conditions where (for unconnected charges) correlations often play a weak role, we will demonstrate that the high connectivity of both polyion species can result in strong correlation effects. To probe the hypothesis that molecular-level connectivity is important, we alter a previous approach of using LS theory to determine local structure in polyelectrolyte systems to incorporate connectivity of the polyions using a PRISM model. ${ }^{62,63,80-82}$

We consider a system with four non-water species, as demonstrated in Figure 1. There are two polymer species, each composed of a chain of charges with valency $z_{P+}$ and $z_{P-}$. Here, we examine the simple case where the valencies are always set to $z_{P \pm}=1$. We only explicitly render the charged moieties as finite-size beads (of size $a_{P+}$ and $a_{P-}$ ), and thus any uncharged polymer link between the two is not explicitly considered as having excluded volume. This chain volume is accounted for separately from the charge-based excluded volume (which implicitly includes ion hydration) in a mean-field contribution described later. The charged beads are spatially correlated at a fixed distance $d_{P+}$ and $d_{P-}$ that sets the spacing between charges. Each charged bead "sees" its neighbors along the chain up to the point at which connectivity correlations are lost. This is effectively the persistence length of the chain, and we approximate this by setting the limit of the connectivity correlations to a number $n_{K}$ away from the original bead (on either side). This is conceptually like having "step function" correlations, with anything less than $n_{K}$ away correlating like a rigid rod and anything more than $n_{K}$ charged species away appearing uncorrelated via chain connectivity. We note that this 'persistence length' is not determined self-consistently, and could in a real system be 
affected by the charged components themselves as well as (for example) excluded volume. The chains have an overall length, $N_{P+}$ and $N_{P-}$, however for the purposes of this work we set $N_{P+}=N_{P-} \rightarrow \infty$. This simplifies the calculations, but does not drastically change the results of this work. In principle it is possible to include variations in chain length $N$ into our studies. For this work we will also retain the assumption that all of the above properties $x=N, n_{K}$, etc. are such that $x_{P+}=x_{P-}=x_{P}$ such that each polymer is the same except for the charge. The same will be true for the ion species, with valencies $z_{+}=z_{-}=z_{ \pm}$and radii $a_{+}=a_{-}=a_{ \pm}$. We could remove this assumption in this theoretical approach, and this is an interesting area for further study. Nevertheless, we start by analyzing a small area of what is an immense parameter space and maintain symmetry between the charged polymers.

The four species are in a given phase with number concentrations $c_{+}, c_{-}$, and $c_{P}$ (the polymer concentration is given as charged monomers per volume). We will consider two phases, shown in Figure 2; a polymer-rich phase $\alpha$ and a polymer-dilute phase $\beta$ that serves as a "bath" of constant chemical potential for the various components. We will therefore specify the quantities associated with the various phases with the appropriate superscript, so for example the concentration of cation in the $\alpha$-phase will be given by $c_{+}^{\alpha}$. For simplicity's sake, we assume that not only does electroneutrality exist in each phase in general but for both the polymer and salt species independently. Therefore, $z_{+} c_{+}^{\alpha}=z_{-} c_{-}^{\alpha}$ and $c_{P,+}^{\alpha}=c_{P,-}^{\alpha}=c_{P}^{\alpha}$, along with the corresponding equations for the $\beta$-phase. This is not necessarily the case; significant experimental work in the past has considered systems where the stoichiometry of the two phases is not the same, either by the concentration of polymer or by adjusting $\mathrm{pH},{ }^{12,13,32,34,35,47}$ leading to decreased stability of the coacervate phase. This represents yet another area of parameter space that may be explored in the future using this model. Nevertheless, if we assume that one of the phases $(\beta)$ is infinitely dilute in polymer (which is the case in the $N \rightarrow \infty$ limit), then so long as the overall stoichiometry of polymer is included in the overall system is electroneutral then this situation will be maintained in the $\alpha$ phase. This will likewise dictate the same condition for the salt stoichiometry, so long as 


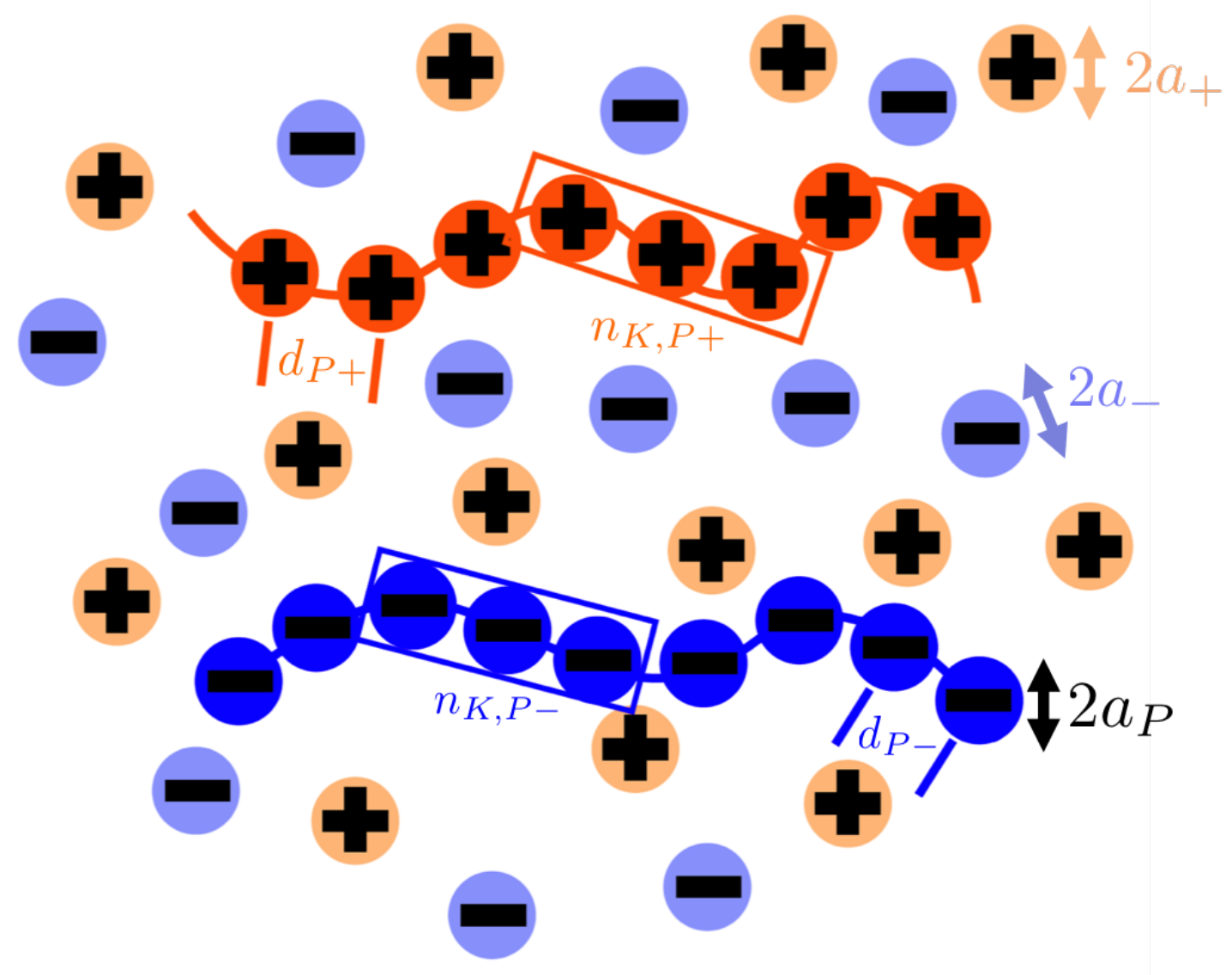

Figure 1: Schematic depiction of the components and their respective parameters for the coacervate model used in this work. For most parameters $\left(d_{P}, n_{K}, a_{ \pm}\right.$, and $\left.a_{P}\right)$, symmetric values will be used for both the polycations and polyanions as well as the cations and anions. This is for the sake of limiting an otherwise large parameter space, while in practice these parameters are not necessarily the same. 


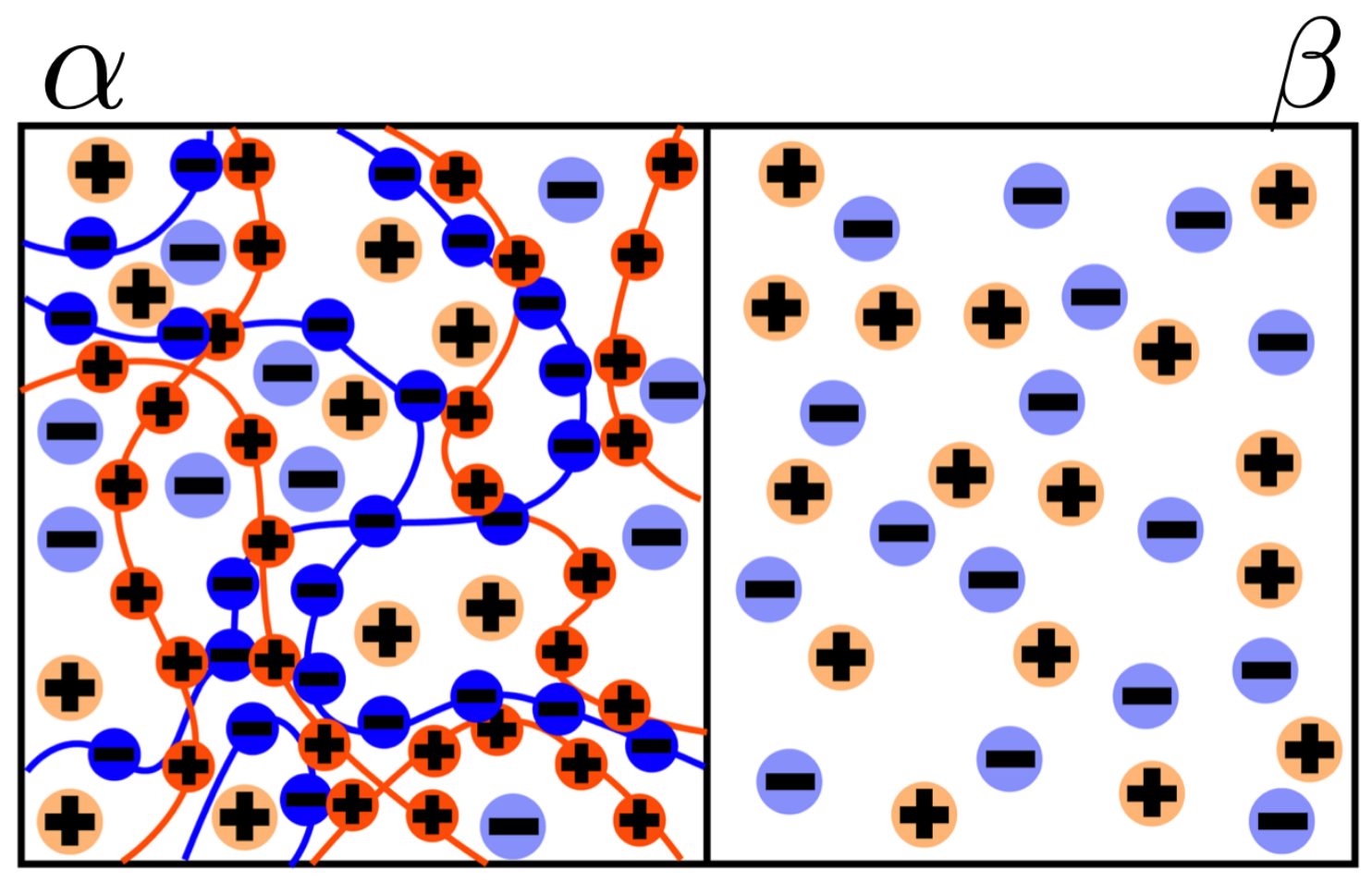

Figure 2: Schematic depiction of a coacervate system considered, consisting of two phases. The polymer-rich $\alpha$ phase contains all of the polyions (due to the infinite chain length) as well as the salt species at a concentration $c_{ \pm}^{\alpha} \neq c_{ \pm}^{\beta}$. The polymer-deficient $\beta$ phase contains only the applied salt concentration $c_{ \pm}^{\beta}$. 
electroneutrality of the overall phases are assumed.

Upon assuming the presence of two phases, we can write the equilibrium conditions for the four species:

$$
\begin{array}{r}
z_{-} \mu_{+}^{\alpha}\left(c_{+}^{\alpha}, c_{-}^{\alpha}, c_{P}^{\alpha}\right)+z_{+} \mu_{-}^{\alpha}\left(c_{+}^{\alpha}, c_{-}^{\alpha}, c_{P}^{\alpha}\right)=z_{-} \mu_{+}^{\beta}\left(c_{+}^{\beta}, c_{-}^{\beta}\right)+z_{+} \mu_{-}^{\beta}\left(c_{+}^{\beta}, c_{-}^{\beta}\right) \\
\mu_{P}^{\alpha}\left(c_{+}^{\alpha}, c_{-}^{\alpha}, c_{P}^{\alpha}\right)=\mu_{P}^{\beta}\left(c_{+}^{\beta}, c_{-}^{\beta}\right)
\end{array}
$$

This set of equations implicitly includes the electroneutrality condition due to the coupling of the $\mu_{+}$and $\mu_{-}$in Equation 2, and the infinite dilution of the $\beta$ phase is implied given the lack of a $c_{P}^{\beta}$ argument in the $\mu^{\beta}$ terms. It is typical to write the chemical potential of the charged ions in the form:

$$
\mu_{ \pm}=\mu_{ \pm, 0}+\mu_{ \pm, e x c}=k_{B} T \ln c_{ \pm}+\mu_{ \pm, e x c}
$$

This form separates the ideal gas contribution to the chemical potential $\mu_{ \pm, 0}$ from the excess chemical potential contribution $\mu_{ \pm, e x c}$. The excess chemical potential $\mu_{ \pm, e x c}$ describes the effect of charge correlations. The equilibrium distribution of ions between the two phases $\alpha$ and $\beta$ will be:

$$
\frac{c_{-}^{\alpha}}{c_{-}^{\beta}}=\frac{c_{+}^{\alpha}}{c_{+}^{\beta}}=e^{\left(z_{-} \mu_{+, e x c}^{\alpha}+z_{+} \mu_{-, e x c}^{\alpha}\right)-\left(z_{-} \mu_{+, e x c}^{\beta}+z_{+} \mu_{-, e x c}^{\beta}\right)}=\lambda\left(c_{+}^{\beta}, c_{-}^{\beta}, c_{P}^{\alpha}\right)
$$

We use the variable $\lambda$ to denote the ratio of salt in and out of the coacervate phase. This $\lambda$ is highly dependent on the concentration of salt that is in the polymer-deficient phase $\left(c_{ \pm}^{\beta}\right)$ and the concentration of polymer in the coacervate phase $c_{P}^{\alpha}$. We set $c_{ \pm}^{\beta}$ as a parameter of the system. $c_{P}^{\alpha}$ will be determined self-consistently with $\mu_{ \pm, e x c}$ and $c_{ \pm}^{\alpha}$, in conjunction with the equilibrium condition on the polymer species (Equation 3). 
The chemical potential of the polymer component is:

$$
\mu_{P}=\mu_{P, 0}+\mu_{P, e x c}=-\ln \left(1-2 \phi_{P}\right)-4 \chi \phi_{P}+\mu_{P+, e x c}+\mu_{P-, e x c}
$$

where $\mu_{P, 0}=-\ln \left(1-2 \phi_{P}\right)-4 \chi \phi_{P}$ is the Flory-Huggins expression for the chemical potential of the polymer in the limit of $N \rightarrow \infty ;^{55}$ this is essentially due to the mixing entropy of the solvent and the short-range energetic penalty for polymer-solvent contacts. This latter term is important if, for example, the backbone is hydrophobic. ${ }^{9}$ This is often the case for most synthetic polymers, and can result in a strong enhancement of the stability of the coacervate. For the purposes of this paper, we will consider only the athermal $\chi=0.0$ situation. The dispersive contributions that $\chi$ is meant to represent will be strongly affected by the highlycorrelated structure of coacervates, making this a non-trivial contribution. ${ }^{83} \phi_{P}=c_{P} \nu_{0}$ is the volume fraction of a given polymer species, and uses the monomer volume $\nu_{0}$. This leads to a mean-field excluded volume associated with the uncharged polymer chain, and is separate from the correlated excluded volume of the charged species. The above form of the chemical potential can be used to write the equilibrium equation:

$$
-\ln \left(1-2 \phi_{P}\right)-4 \chi \phi_{P}=\mu_{P+, e x c}^{\alpha}+\mu_{P-, e x c}^{\alpha}-\mu_{P+, e x c}^{\beta}-\mu_{P-, e x c}^{\beta}=-\Delta \mu_{P}\left(c_{+}^{\beta}, c_{-}^{\beta}, \lambda, c_{P}^{\alpha}\right)
$$

where we have defined a $\Delta \mu_{P}=\sum \mu_{P, e x c}^{\beta}-\sum \mu_{P, e x c}^{\alpha}$ that is the difference in excess chemical potential between the two phases. We have assumed that larger polymer-conformational behaviors are not significant, which is in line with scattering results demonstrating Gaussian chain behavior in the coacervate. ${ }^{84}$ The $\phi_{P} \rightarrow 0$ limit retains the classical Flory-Huggins result $\mathrm{n}$ the absence of the correlation-based excess chemical potential. ${ }^{55}$ The terms in this equation are a function of the concentration of the polymers as well as the salt concentration (via the $\Delta \mu_{P}$ term), and both equations share a common unknown $c_{P}^{\alpha}$. Solving for this yields the equilibrium concentration of the coacervate phase. There is always one solution to these equations at $c_{P}^{\alpha}=0$, which is the trivial case that there is no coacervate formation; 
however, there is often a second set of conditions that fits these conditions that corresponds to the formation of a coacervate phase for a given value of $c_{+}^{\beta}$ and $c_{-}^{\beta}$; this formation is an equilibrium phase if the free energy is lower than the non-coacervate state.

This sets up the thermodynamic description of the system, which dictates the formation or non-formation of a coacervate phase. The form of the $\mu_{x, \text { exc }}($ where $x=+,-, P$ ) remains to be determined, however. For this we rely on Liquid State theory (LS) calculations that account for the local geometry described at the beginning of this section. ${ }^{74}$ In particular, the use of the Reference Interaction Site Model (RISM) or the related Polymer-RISM (PRISM) model can provide the ideal combination of local order information and subsequently largescale thermodynamic parameters. ${ }^{74,80}$ This approach can provide an alternative to perturbation theories; namely, while perturbation approaches tend to truncate a series expansion at some finite term and cannot always converge, ${ }^{58}$ LS theories take these expansions to infinite terms and then compare them to develop what are known as "closure relationships." 74,80 These comparisons are typically not exact, however the resulting approximation is vastly different than a perturbation-type approximation. ${ }^{74}$ In situations where perturbation theories tend to not converge (such as highly-correlated charged systems) the approximation is better but nevertheless opaque - certain classes of "diagrams" are neglected, and an understanding of the physical ramifications of this neglect is still poorly developed. ${ }^{58,74}$ Nevertheless, this class of theories provides excellent and often-quantitative matching of structural information to both simulation and experiment. ${ }^{74,80}$

The PRISM approach we take requires the iterative solution of two equations; the PRISM formulation of the fundamental Ornstern-Zernicke (OZ) equation and the Debye-Hückel Extended Mean Spherical Approximation (DHEMSA) closure relationship that has been recently developed. ${ }^{74,79,80}$ The PRISM version of the OZ equation is:

$$
\hat{h}_{i j}=\hat{\omega}_{i k} \cdot \hat{c}_{D, k l} \cdot \hat{\omega}_{l j}+c_{l} \hat{\omega}_{i k} \cdot \hat{c}_{D, k l} \cdot \hat{h}_{l j}
$$


where hats denote Fourier-transformed values. ${ }^{74,80}$ The overall correlation between species $i$ and $j$ is given by $h_{i j}$. This occurs either through a direct interaction, which is captured by the direct correlation function $c_{D, i j}$, or via an indirect interaction that is a convolution of $c_{D, i j}$ and $h_{i j} .{ }^{74}$ Diagrammatic expansions of this equation are used as the starting point for the development of LS theory, ${ }^{74}$ and the inclusion of the intramolecular correlation function $\omega_{i j}$ includes the correlation information between sites on the same molecule. In PRISM theory, this specifically expresses the molecular structure along the polymer backbone. ${ }^{74,80}$ The correlation functions $c_{D, i j}$ and $h_{i j}$ are matrix quantities where each index can be any one of the distinct species in the system. For our system, these correspond to the,,$+- P+$, and $P$ - species. For each pair of components $i$ and $j$, there are now two unknowns: the correlation functions $c_{D, i j}$ and $h_{i j}$. In order to determine these, we require a further closure relationship linking the two values. For our system we use the DHEMSA closure relationship developed by Zwanikken, et al.. ${ }^{79}$ This is a piecewise definition of the various correlation functions: 79

$$
\begin{array}{r}
h_{i j}(r)=-1.0 \text { if } r<2 a \\
c_{D, i j}(r)=h_{i j}^{D H}-\ln \left(1+h_{i j}^{D H}\right)-\beta u_{i j} \text { if } r>2 a
\end{array}
$$

where $-\ln \left(1+h_{i j}^{D H}\right)= \pm \beta \phi_{++}+\phi_{H S}$ sets a reference correlation function $h_{i j}^{D H}$ that is based on the solution of the Poisson-Boltzmann equation in radial coordinates: ${ }^{79}$

$$
\beta \nabla^{2} \phi_{++}(r)=\kappa^{2} \sinh \beta \phi_{++}(r)
$$

with the boundary conditions: ${ }^{79}$

$$
\left(\frac{\partial \phi_{++}}{\partial r}\right)_{r=2 a}=-\frac{\lambda_{B}}{4 a^{2}}
$$


and

$$
\left(\frac{\partial \phi_{++}}{\partial r}\right)_{r \rightarrow \infty}=0
$$

where $\lambda_{B}$ is the Bjerrum length $\lambda_{B}=e^{2} /\left(4 \pi \epsilon k_{B} T\right)$ and $\kappa$ is the inverse Debye length $\kappa=$ $\sqrt{8 \pi \lambda_{B} \rho_{+}} \cdot{ }^{79}$ In this overall calculation, there are only a small number of inputs that are needed: $u_{i j}=\lambda_{B} z_{+} z_{-} / r$ is the potential between charges $i$ and $j$, and the densities $\rho_{x}\left(x=\rho_{+}, \rho_{-}, \rho_{P}\right)$ are determined by the overall thermodynamics. We refer the reader to the paper by Zwanikken, et al. for a thorough discussion of the nature of this closure in the non-PRISM context. ${ }^{79}$ In using PRISM, we must also dictate the behavior of $\omega_{i j}$ that captures the intramolecular correlations. We approximate $\omega_{i j}$ with the expression for a rigid rod: ${ }^{85}$

$$
\hat{\omega}_{i j}=\delta_{i j}+\delta_{i j} \delta_{i P} \sum_{l=1}^{n_{K}} \frac{2\left(n_{K}-l\right)}{n_{K}} \frac{\sin \left(l k d_{P}\right)}{l k d_{P}}
$$

This expression treats each non-polymer species as a $\delta$-function (such that there are appropriately no intramolecular correlations), while the polymer species are connected directly to $n_{K} \times 2$ other polymers species of the same type. This approximates a semiflexible polymer, where each monomer is connected to charges that are placed rigidly nearby, however charges distant along the chain contour become uncorrelated. $n_{K}$ thus sets the extent that this rigidity extends along the polyelectrolyte backbone. This simplified representation considers all chain segments to be in the middle of the chain (its neighbors are always present), which is appropriate for the $N \rightarrow \infty$ approximation we are making. ${ }^{80}$ Future improvements to the current method are possible if the value of $\hat{\omega}_{i j}$ is rendered self-consistent with the correlations. This is often accomplished via molecular simulation. ${ }^{77,85,86}$

We note that the use of the DHEMSA closure approximation in the scope of the PRISM version of the $\mathrm{OZ}$ equation is an approximation; even though the PRISM equation includes connectivity, the closure relationship itself does not. This is known to be an important feature of widely-used PRISM closures, in particular the Laria-Wu- 
Chandler (LWC) closure that is known to be effective for polyelectrolyte systems. ${ }^{75,76,78,88,89}$ We use the DHEMSA closure instead of the LWC, primarily due to the ease with which DHEMSA converges over a wide array of polyelectrolyte and salt concentrations. ${ }^{79}$ Since we are interested in qualitative results, and DHEMSA is known to be in excellent agreement with simulations outside of PRISM, ${ }^{79}$ we do not expect drastically unrealistic results. However, the use of more precise closure relationships represents an area for further refinement of the theory. In order to understand the result of this method, we invoke the similarity between DHEMSA results and those for the Hypernetted Chain (HNC) closure relationship. This is known to be the case in most regimes of interest. ${ }^{79}$ We would like to, however, use this similarity to permit us to use the HNC-based expression for the excess chemical potential: ${ }^{74}$

$$
\frac{\mu_{i, e x c}}{k_{B} T}=\sum_{j} \frac{c_{j}}{2} \int h_{i j}\left[h_{i j}-c_{D, i j}\right] d \mathbf{r}-c_{j} \int c_{D, i j} d \mathbf{r}
$$

By coupling the calculation of the complete set of correlation functions $h_{i j}$ and $c_{D, i j}$ to the values of $\mu_{i, e x c}$, we have a way of coupling the local information calculated via the PRISM theory to a thermodynamic value that can be used in the thermodynamic calculation outlined in the first part of this section.

Improvements to the formalism are possible; for example, the use of the DHEMSA with PRISM may be evaluated in the future in comparison with simulation results, ${ }^{74}$ or the LWC closure can be used instead of the DHEMSA closure. ${ }^{89}$ The use of the highlysimplified hard rod version of $\hat{\omega}$ may be likewise reevaluated, where instead of an approximate description of a semiflexible chain more accurate correlations are taken from simulation. ${ }^{77,86}$ Such investigations will permit a more thorough understanding of the role of chain flexibility. Furthermore, the assumption of $N \rightarrow \infty$ may be relaxed in the future to deal with chains that are relatively short. Nevertheless, we expect this method to provide a new glimpse into the effects of local structure on the thermodynamics of complex coacervation that is orthogonal 
to many of the current methods considered in the literature. This method uniquely considers the thermodynamics in a way that is related directly to local structure, while current models have a difficult time (at best) in understanding these structural correlations. ${ }^{7,8,66,67,71,72}$ This may even provide a glimpse into biologically-relevant binding behaviors where local charge organization is implied by the precise placement of charged moieties on proteins. ${ }^{44}$

\section{Results}

\section{Coacervate Structure}

PRISM has the ability to articulate local charge structure in a fashion such that local correlations are directly demonstrable. The results of the calculation can provide predictions of the pair correlation function $g_{i j}(r)=h_{i j}(r)+1$, which is between all of the species $i$ and $j$. Figure 3 demonstrates a characteristic pair correlation function for a system that is both in the coacervate phase and in the dilute phase for a symmetric monovalent sys-

tem. For each system, there are six possible curves; polycation-cation, polyanion-cation, cation-cation, cation-anion, polycation-polycation, and polyanion-polyanion (the opposite combinations are the same so long as charged species are symmetric in valency and size).

Features of the correlation functions directly correspond to certain types of charge structures. In Figure 3, we can start to assign the peaks accordingly; we introduce a schematic in Figure 3e that labels the relative locations between charged species and indicates the peaks that are seen in the correlation functions in Figure 3b. The primary species are indicated in bold colors, while lighter colors indicate the correlations via connectivity that has been imposed via the PRISM calculation. This connectivity leads to a large amount of local structure, with distant correlation peaks being prevalent even in the symmetric monovalent case. These types of features, such as oscillatory behavior or connectivity-induced peaks, are unrealizable with traditional Poisson-Boltzmann theory ${ }^{58}$ and typically require ad-hoc theoretical approximations such as charge renormalization ${ }^{90}$ to enable a description of the 
thermodynamics. While these renormalization (or related) methods are often powerful, we can reproduce the entire correlation behavior within the scope of the primitive model in a fashion that requires far fewer assumptions about the system.

It is these correlations that characterize complex coacervation; there is a large set of peaks that correspond to the abundance of oppositely-charge chains adjacent to each other that are more pronounced than the oppositely-charged single ions peaks. This is due to cooperativity; the connectivity of adjacent charges reduces the entropic penalty of bringing two oppositely-charged monomers together if their adjacent charges are likewise next to each other. This connectivity effect is particularly significant even at large salt concentrations $c_{ \pm}^{\beta}$, where there is little driving force for phase separation otherwise. Coacervates are typically stable up to salt concentrations on the order of $1-5 \mathrm{M},{ }^{34-38,48}$ where the salt charges alone take up $\sim 10 \%$ of the volume in the system rendering it a relatively dense sea of ions. Associations between unconnected charged species rapidly decay in such systems (Figure 3d, red and black curves). We demonstrate the connectivity effects in Figure 3a-d, which demonstrates the correlation behavior as a function of salt concentration $c_{ \pm}^{\beta}$. As the salt concentration increases, the cation-anion correlations decrease significantly. However, the polycation-polyanion system maintains peaks that are associated with connectivity correlations well beyond the correlation distances observed in their non-connected counterparts. This drives the system towards polymer-dense states, since even at high salt concentration connectivity increases the correlations between opposite charges.

\section{Phase Behavior and Counterion Release}

In order to evaluate the coupled sets of Equations 5 and 7 , we set the unknown values $c_{+}^{\beta}$, $c_{-}^{\beta}$ to the desired external salt concentration. We can then set the value of $c_{P}^{\alpha}$, and then calculate a value of $\Delta \mu_{T O T}$ given by the equation:

$$
-\ln \left(1-2 \phi_{P}^{*}\right)-4 \chi \phi_{P}^{*}+\Delta \mu_{P}\left(c_{+}^{\beta}, c_{-}^{\beta}, \lambda, c_{P}^{\alpha, *}\right)=\Delta \mu_{T O T}\left(c_{ \pm}^{\beta}, c_{P}^{\alpha, *}\right)
$$



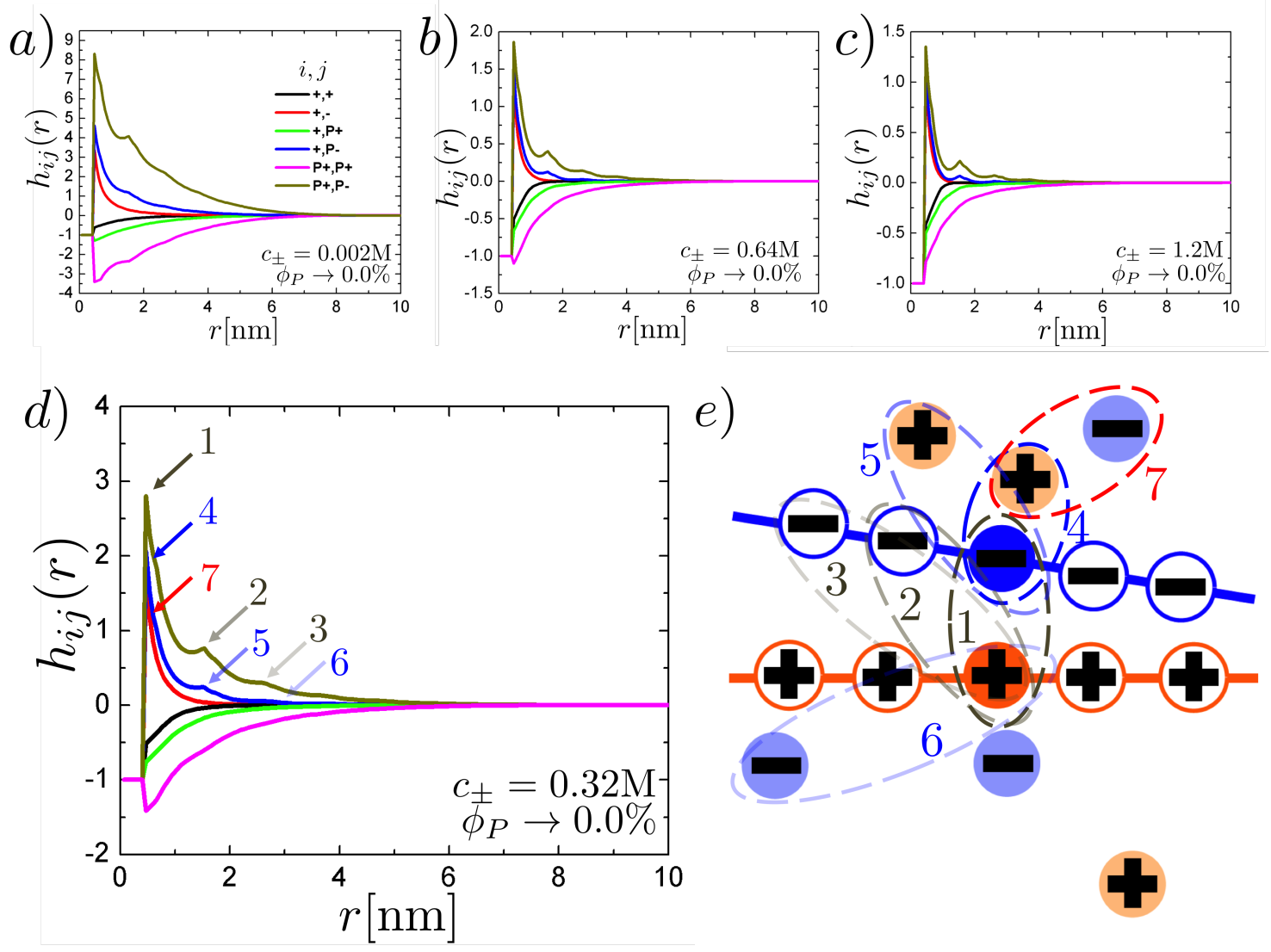

Figure 3: (a-d) Correlation functions $h_{i j}$ between each pair of components for $a_{P}=0.2 \mathrm{~nm}$, $a_{ \pm}=0.2 \mathrm{~nm}, d_{P}=0.5 \mathrm{~nm}$, and $n_{K}=6$. We note that $h_{i j}(r)$ is technically supposed to be $h_{i j}(r)>-1$, and the DHEMSA closure provides unphysical values. The literature suggests that even with this artifact, the calculation of quantities such as $\mu_{j}$ still provide reasonable results due to their reliance on differences between $h(r)$ values rather than absolute values of $h(r) .{ }^{79}$ We note that $h_{i j}(r)=g_{i j}(r)+1.0$. (e) Dashed ovals and corresponding numbers encircle prominent correlations that are observed in these calculations. They correspond to similarly-colored arrows and numbers denoting peaks in (d). Brown-colored ovals (1-3) represent polymer-polymer correlations, blue ovals (4-6) represent polymer-ion correlations, and the red oval (7) represents an ion-ion correlation. Strong colors are for nearby correlations, while light colors are for distant correlations. Original charges along the polymer chain are filled, while connected charges are open. 
where $c_{P}^{\alpha, *}$ is a set value of $c_{P}$ that is not necessarily at equilibrium. Based on Equation 7 , equilibrium will be obtained when $\Delta \mu_{T O T}=0$. The value of $\lambda$ can be determined as if the salt were in equilibrium, and thus for a given $c_{P}^{\alpha, *}$ only the polymer volume fraction is kept out of equilibrium while the distribution of charges $\lambda$ equilibrates based on the given constraints. This enables the calculation of an energy landscape via the integration of the chemical potential difference $\Delta \mu_{T O T}\left(c_{ \pm}^{\beta}, c_{P}^{\alpha, *}\right)$ :

$$
\tilde{f}\left(c_{ \pm}^{\beta}, c_{P}^{\alpha}\right)=\int_{0}^{c_{P}^{\alpha}} \Delta \tilde{\mu}_{T O T}\left(c_{ \pm}^{\beta}, c_{P}^{\alpha, *}\right) d c_{P}^{\alpha, *}
$$

where tildes represent normalization by thermal energy $k_{B} T$. Physically, this represents slowly increasing the polymer concentration in a coacervate phase that has salt in equilibrium with a bath. In Figure 4a we plot a series of these free energy landscapes as a function of the concentration $c_{P}^{\alpha}$ for a number of different values of $c_{ \pm}^{\beta}$ and a monovalent salt. These free energy curves at intermediate salt $c_{ \pm}^{\beta}$ can have one of two minima. A minimum value at $c_{P}^{\alpha}=0$ corresponds to a dilute solution that is resistant to coacervate formation, and in Figure $4 \mathrm{a}$ this is observed for the $c_{ \pm}=0.18 \mathrm{M}$ curve. Alternatively, a minimum at $c_{P}^{\alpha}>0$ is a coacervate phase that is often lower in free energy and thus the observed state (for example, the $c_{ \pm}=0.13 \mathrm{M}$ curve in Figure 4a). Usually, as salt $c_{ \pm}^{\beta}$ is increased the free energy landscape tilts up such that this coacervate phase is at a higher energy (however still a local minimum). Ultimately, the local minimum itself becomes unstable. This free energy landscape picture is characteristic of a first-order phase transition. ${ }^{91}$ We hypothesize that the first-order transition (as opposed to a second order transition as is expected in mean field theories $)^{7,8,57}$ is conceptually related to the competition between local ordering of oppositelycharged polyions and the cooperative electrostatic energy. Experimental data has shown sharp transitions characteristic of first-order transitions, ${ }^{34}$ however it is unclear that these are truly first-order. This aspect will require further interrogation, both experimentally and theoretically. 

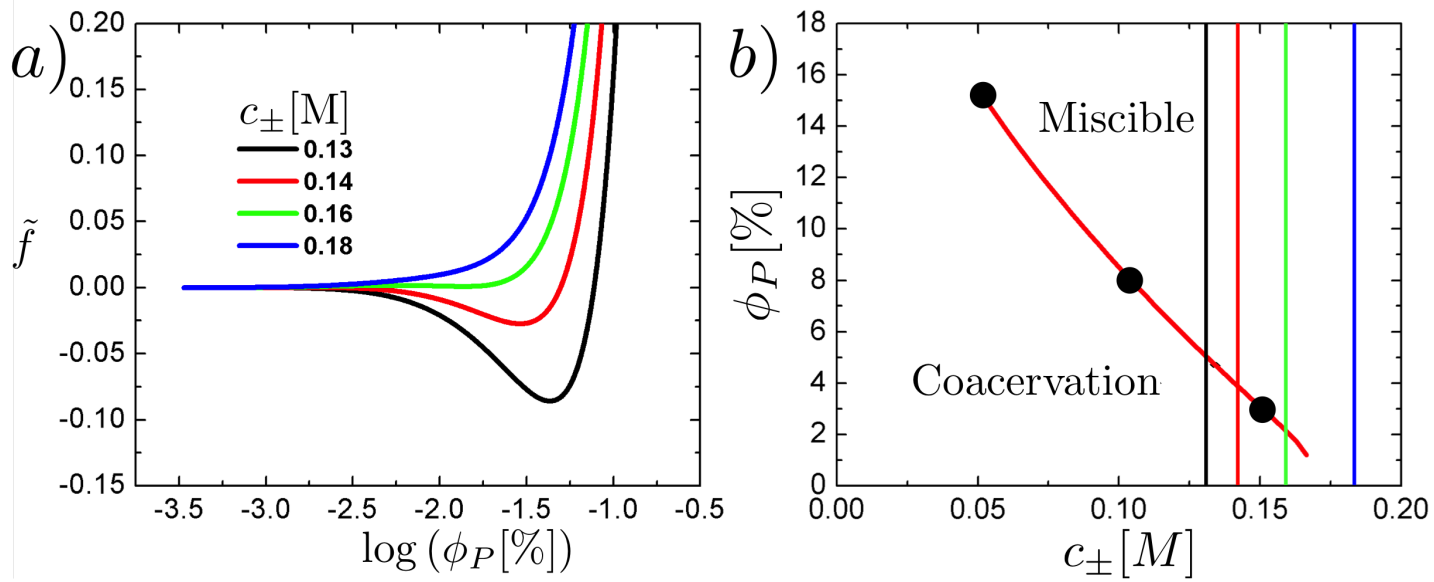

Figure 4: (a) Free energy landscapes show that at as the salt concentration is decreased a free energy minimum occurs at finite polymer concentration. (b) Landscapes in (a) result in the phase boundary for coacervation in the $\phi_{P}$ (percent volume occupied by polymer) versus $c_{ \pm}$(salt concentration) plane. Coacervation occurs on the bottom left hand corner of the graph. $a_{P}=0.2 \mathrm{~nm}, a_{ \pm}=0.2 \mathrm{~nm}, d_{P}=0.5 \mathrm{~nm}$, and $n_{K}=6$. Colored lines denote locations of the corresponding free energy landscape for a number of different salt concentrations $c_{ \pm}$ as a function of $\log$ salt concentration $\log \left(\phi_{P}\right)$ plotted in (a).Black dots in (b) correspond to locations where we will plot correlation functions in Figure 5.

With this free energy picture, we calculate in Figure $4 \mathrm{~b}$ a phase diagram in the $c_{ \pm}^{\beta}-c_{P}^{\alpha}$ space, which has been experimentally measured for a few systems. ${ }^{34}$ This shows the general trend that increasing salt concentration $c_{ \pm}$leads to a decrease in the volume fraction of polymer $\phi_{P}$ in the polymer-rich phase until the coacervate disappears (around $c_{ \pm}=0.17 \mathrm{M}$ in the current example). Within the simple representation of the phase boundaries, we can probe the local structure via the PRISM results used to generate the graph. We show this in Figure 5, which plots in (a-c) the black points in Figure 4b and in (d-e) plots the $\beta$-phase correlations for these same points. These correlation functions $h_{i j}(r)$ demonstrate a tradeoff between bound ions in the $\beta$-phase (bottom row) and strong oppositely-charged polyions in the $\alpha$-phase, suggesting interpretation via the counterion release mechanism of coacervation formation. ${ }^{44,52}$ In particular, we emphasize the difference in the polyion-ion correlations versus the ion-ion correlations for oppositely charged species. In the bottom row ( $\beta$ phase), there is a significant abundance of ions next to the polyion compared to next to a salt ion, 

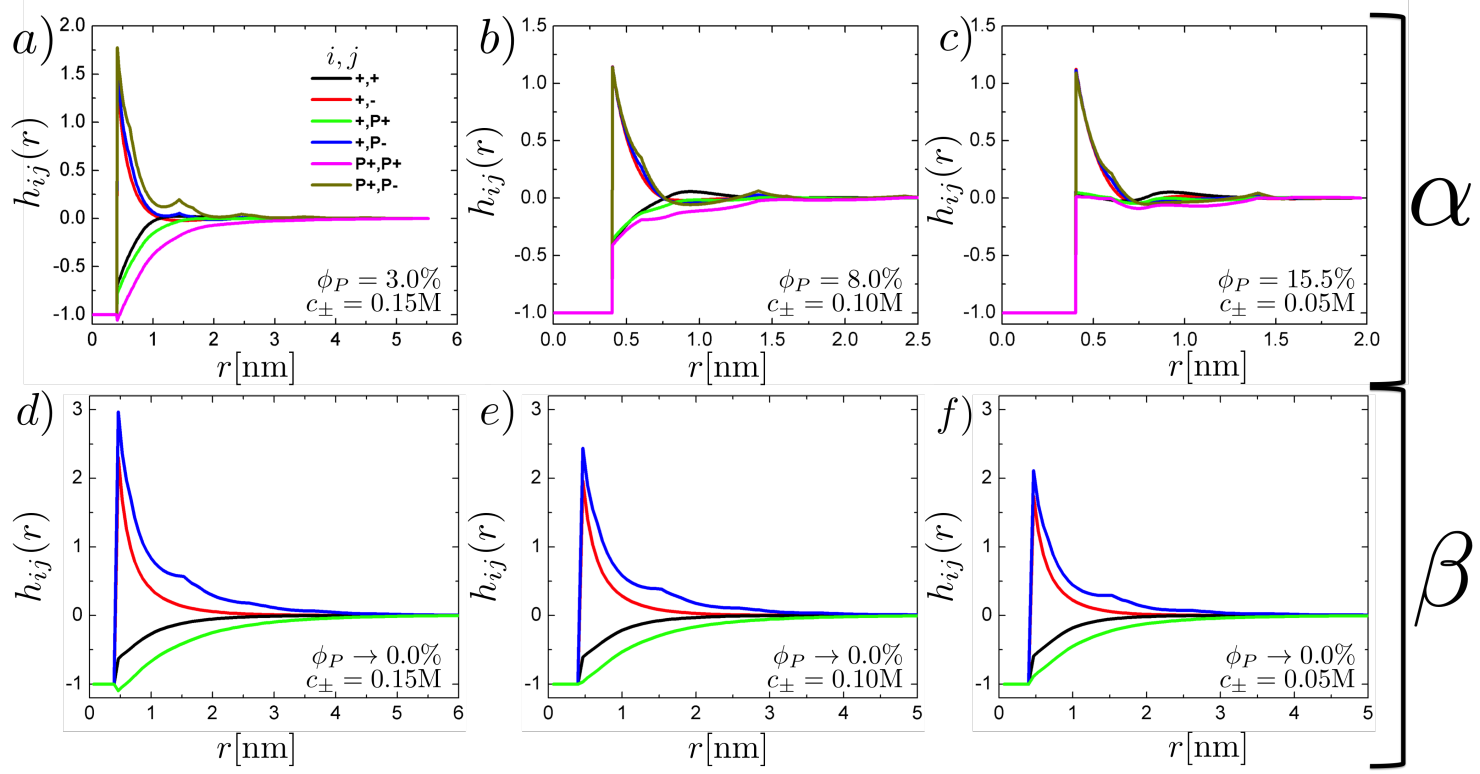

Figure 5: (a-c) Correlation functions $h(r)$ for the black points in Figure 4b. (d-f) Correlation functions for the corresponding $\beta$-phase equivalent to the $\alpha$-phases in (a-c) respectively. We note the important tradeoff in molecular features; The blue $(+, \mathrm{P}-)$ and green $(+, \mathrm{P}+)$ curves are nearly indistinguishable from the red $(+,-)$ and black curves $(+,+)$ respectively in $(\mathrm{a}-\mathrm{c})$, where the ions do not care whether they are near a polyion or an oppositely-charged salt ion. Instead, strong correlations are primarily observed for the complexation between oppositelycharged polyions (olive and magenta curves, $\mathrm{P}+, \mathrm{P}+$ and $\mathrm{P}+, \mathrm{P}-$ ). The blue and green curves demonstrate a much stronger correlation effects than the red and black curves in (d-f); they represent the strong ion correlations around a polyion monomer where opposite charges are bound strongly (blue and green) when compared to the salt ions not near a polymer (red and black). This represents the local structural manifestation of the "counterion release" mechanism that leads to coacervate formation. We note that $h_{i j}(r)=g_{i j}(r)+1.0$. 
which is indicative of counterion condensation. In the top row ( $\alpha$ phase), there is very little difference between these correlations, indicating that the salt ions do not distinguish between salt and polyions (i.e. the counterions have been 'released'). This theory therefore captures the conceptual "counterion release" argument, representing one prevailing understanding of coacervate formation.

The phase diagram in Figure 4b can be used to demonstrate trends due to experimentally observable changes in the system. This initial case considers $z_{-}=z_{+}=1, n_{K}=6, d_{P}=$ $0.5 \mathrm{~nm}$, and $a_{+}=a_{-}=a_{P}=0.2 \mathrm{~nm}$, which means that all charged species are identical in size and charge. This system will serve as the baseline for comparing the effects of different parameters such as ion size, polymer connectivity, and polymer excluded volume. These choices are motivated by typical values seen in experimental systems. For example, this charge size $a_{ \pm}$is on the order of magnitude of the size of hydrated ions in solution, ${ }^{81}$ and the choice of $n_{K}$ is consistent with a typical Kuhn length for a semiflexible polymer. ${ }^{93}$

\section{Relationship to Debye-Hückel theory}

Beyond exhibiting the classical "counterion release" concepts, the theory developed in this manuscript also reproduces Debye-Hückel theory in the appropriate limits. Specifically, this limit should be retained upon (1) diluting the system, (2) decreasing the excluded volume, and (3) removing connectivity of the charges. The second and third limits are those previously discussed as representing the neglected assumptions of Debye-Hückel based Voorn-Overbeek approaches.

The Voorn-Overbeek theory utilizes the electrostatic contribution to the chemical potential:

$$
\mu_{P}=\mu_{ \pm}=\frac{\kappa \lambda_{B}}{2(1+\kappa a)}
$$

into the aforementioned calculation (where we would use the PRISM theory results). We can 


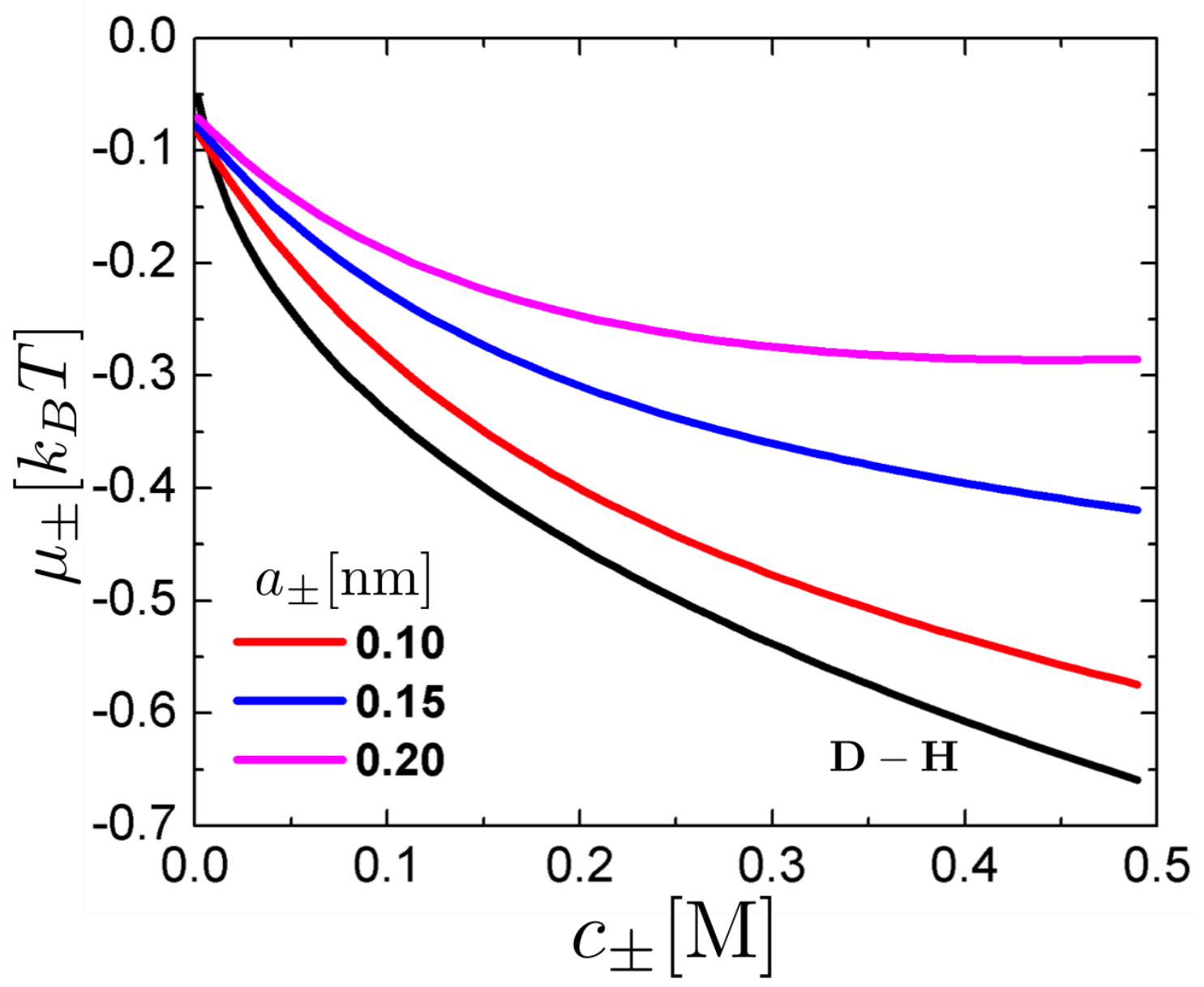

Figure 6: In Voorn-Overbeek theory, the excess chemical potential $\mu_{ \pm}$follows the black curve (the Debye-Hückel, D-H result) as salt concentration $c_{ \pm}$is changed. The current model approaches this behavior the limit of small $a_{ \pm}$and $a_{P}=0.1 \mathrm{~nm}$ (red curve). However, $\mu_{ \pm}$quickly moves away from this limiting result as charges are increased $a_{ \pm}>0.1 \mathrm{~nm}$ (blue, magenta curves). 
show that at the appropriate limit leads to appropriate values; when $a_{ \pm} \rightarrow 0, a_{P} \rightarrow 0$, and $n_{K}=1$ (no connectivity) we can calculate using LS theory the value of $\mu_{P}=\mu_{ \pm}$as a function of concentration and compare it to the above equation. This is plotted in Figure 6, which demonstrates that in the appropriate limit (low concentration $c_{P}, c_{ \pm} \rightarrow 0$, small $a_{P}, a_{ \pm}$) this result is indeed obtained. However, even upon retaining the $n_{K}=1$ condition we increase to $a_{P}=0.2 \mathrm{~nm}$ and $a_{ \pm}=0.2 \mathrm{~nm}$ and find that this has the effect of significantly changing the value of $\mu_{P}$, especially at high $c_{P}$. Excluded volume thus plays a significant role, even without connectivity effects.

We demonstrate how the connectivity further changes (in a drastic way) the values of the chemical potentials $\mu_{P}$ and $\mu_{ \pm}$. We plot in Figure $7 \mathrm{a}$ and $\mathrm{b}$ the calculation of the excess chemical potential for $\mu_{P, e x c}\left(\phi_{P}, c_{ \pm}\right)$and $\mu_{ \pm, e x c}\left(\phi_{P}, c_{ \pm}\right)$which are both functions of both salt and polyion concentration. These landscapes can be compared to the same values for the Debye-Hückel excess chemical potential $\mu_{e x c, D-H}$ in Figure $7 \mathrm{c}$, obtained directly from Equation 17. The excess chemical potential in the Debye-Hückel case becomes more and more negative as the density of ions and/or density of polyions is increased. This is due to the increasing electrostatic attraction. Alternatively, our PRISM-based model for both the polyion and salt species have very different trends in Figure 7a and b. The low- $\phi_{P}$ and low$c_{ \pm}$region looks similar, with an initial decrease in the excess chemical potential. $\phi_{P}$ initially shows a sharp decrease in the excess chemical potential due to cooperative behaviors that initially enhance coacervation. However, as $\phi_{P}$ and/or $c_{ \pm}$are increased, there is an increase in excluded volume effects due to the decreased configurational entropy enforced by the finite size of the species. The strong differences are apparent, for example at low polymer fraction $\phi_{P}=0.04 \%$ all excess chemical potentials are plotted together in Figure $7 \mathrm{~d}$, where the Voorn-Overbeek (Debye-Hückel) and PRISM models can be directly compared. The results are striking, since despite approaching the Debye-Hückel limit in the salt ions there is no similar limit for the polyions. This is not surprising, since the connectivity has long been known to prevent the realization of a sufficiently low concentration appropriate for the 
Debye-Hückel limit. ${ }^{69}$
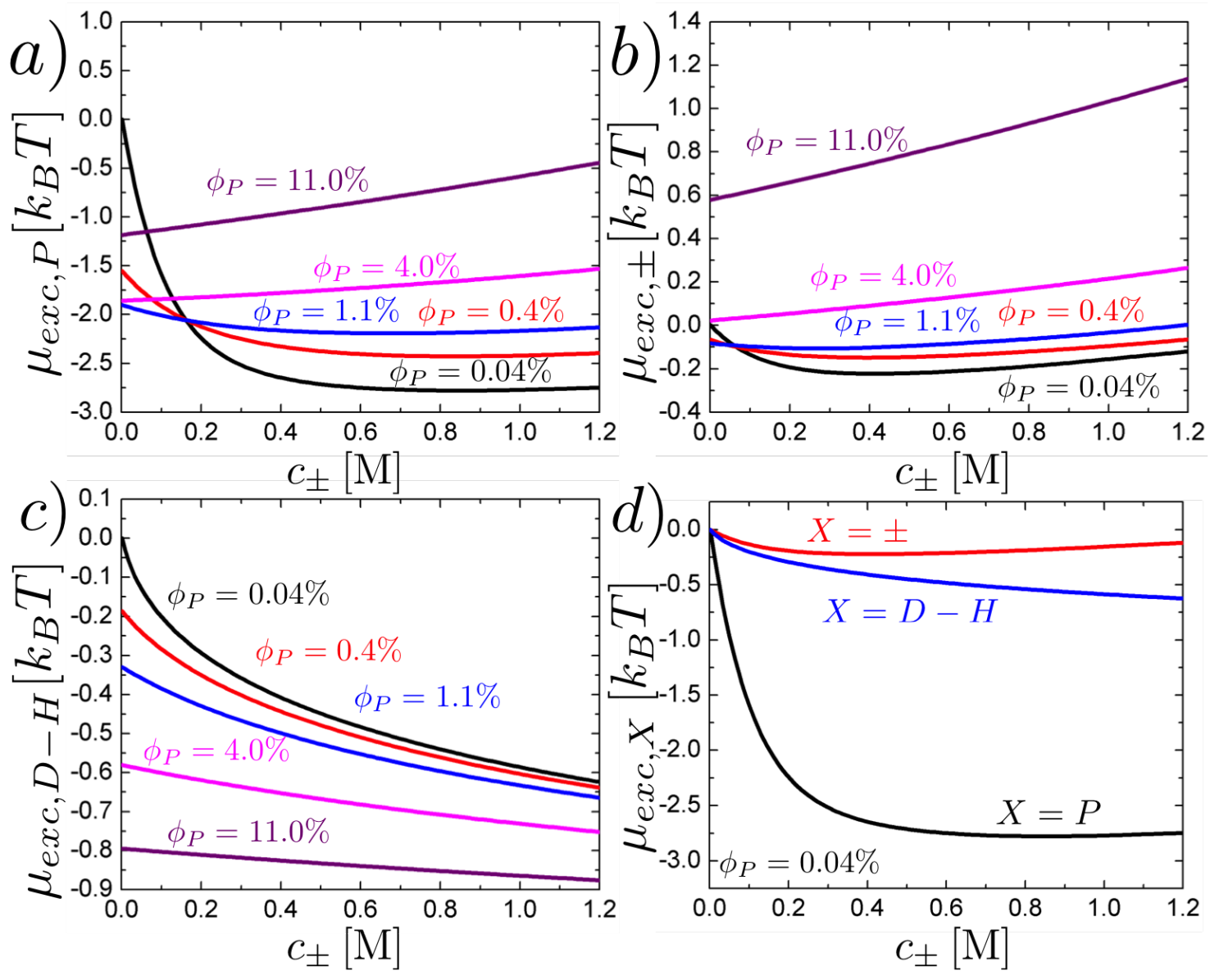

Figure 7: Excess chemical potential $\mu_{e x c, X}$ as a function of salt concentration $c_{ \pm}$and polymer volume fraction $\phi_{P}$ for polyion $X=P$ (a) and salt $X= \pm(\mathrm{b})$. The complicated interplay of the different landscapes can be contrasted to the straightforward landscape of the DebyeHückel expression for the excess chemical potential $\mu_{e x c, D-H}$ that is plotted in (c). For a direct comparison, $\mu_{e x c, X}$ is shown in (d) for $X=P, \pm$, and $D-H$ for the dilute-polymer situation. In the appropriate limit, the ion excess chemical potential retains the Debye-Hückel result. The polymer species does not, due to its connected nature $n_{K}=6$ prevents the dilute limit from being realized. We note that in (a) and (b) there are significant upturns in the excess chemical potential due to excluded volume; this is not present in the Debye-Hückel result.

We note that, despite reproducing the Voorn-Overbeek and counterion release concepts in the appropriate limits, the coacervate system initially shown is such that the critical salt concentration is an order of magnitude below that of many experimental systems. It is therefore important to interrogate the differences between this model and traditional VoornOverbeek and related theories, ${ }^{7-9,57,66,67}$ and subsequently examine how the articulation of 
molecular features may strongly alter the thermodynamics of coacervation. We identify two specific features within the scope of this model that strongly affect coacervation behavior, and demonstrate how this model relates to classical approaches. In particular, most theories specifically neglect both excluded volume and connectivity correlations, and both of these

effects have strong but opposing ramifications in the context of coacervate thermodynamics.

\section{Charge Connectivity}

Charge correlations enforced by chain connectivity are neglected from the classical VoornOverbeek theory, however Figure 5 demonstrates that strong differences in the pair correlation functions arise as a result. As two charges on oppositely-charged polyions interact, their adjacent charges along the chain are highly likely to interact. This interaction is less entropically unfavorable than the initial pair of ions, since there is significantly less penalty in translational entropy for the neighboring charges to interact. To probe this effect, we can change connectivity in our model by adjusting one of two parameters $-n_{K}$ and $d_{P}$. The value of $n_{K}$ changes the effective "stiffness" of the chain by determining the scope of the connectivity correlations along the chain. At $n_{K}=1$, there are no correlations and each charged polyion species is identical to a salt ion (besides potentially the size of the charges). As $n_{K}$ is increased, the position of charges are fixed at a distance $\left(n_{K}-1\right) d_{P}$ such that the original charge senses neighbors along the chain for a total number of $n_{K}-1$ charges. After that, connectivity correlations are considered negligible. We demonstrate the effect of this in Figure 8a, which plots the phase coexistence for coacervates in the $c_{P}$ vs. $c_{ \pm}$ plane for $n_{K}=1-10$ at constant $a_{P}=0.2 \mathrm{~nm}, a_{ \pm}=0.2 \mathrm{~nm}$, and $d_{P}=0.5 \mathrm{~nm}$. As $n_{K}$ is increased, connectivity correlations are enhanced and consequently coacervate formation is enhanced. This is a drastic effect, with the critical salt concentration being increased to very large values. This provides a direct connection with the strength of coacervation and the molecular features of charge connectivity, suggesting that design at a molecular level of coacervate materials is possible. In Figure 8 the ratio of charges in the $\alpha$ versus $\beta$ phase, 
$\lambda$, is demonstrated by the dashed lines. We note that in our theory the number of salt ions is typically lower in the polymer-rich phase, and decreases as the concentration of polymer increases.
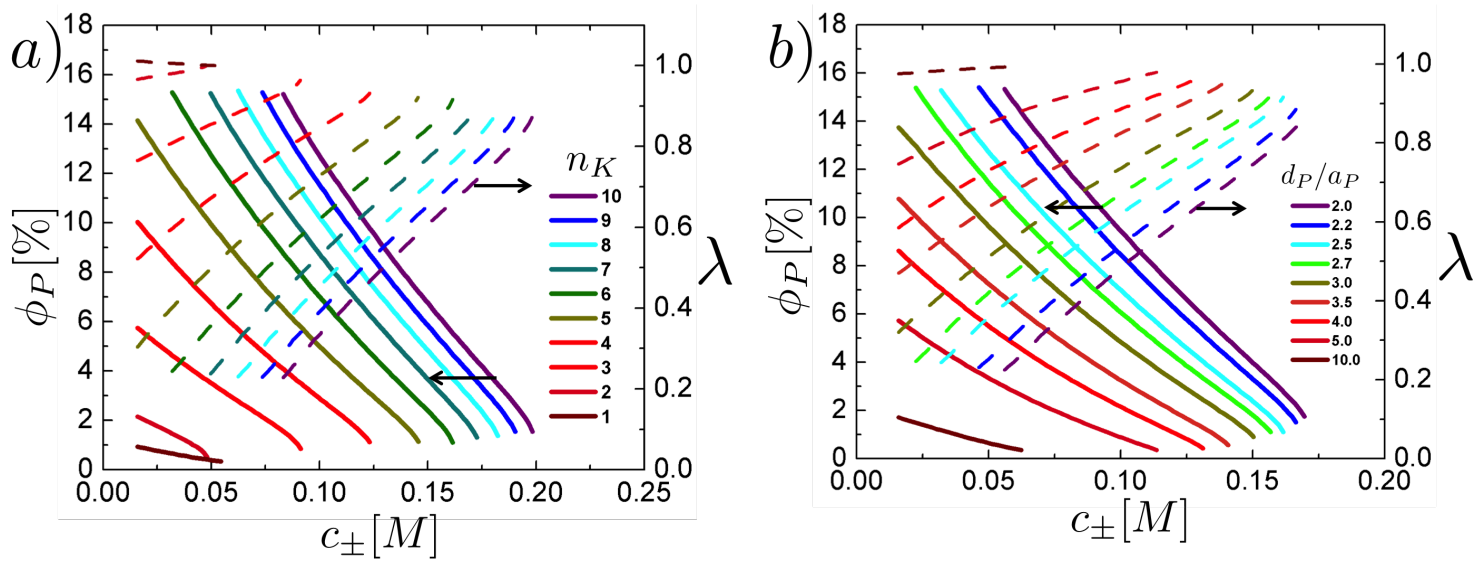

Figure 8: (a) Phase boundaries (solid lines, left axis) and ion partitioning $\lambda$ (dashed lines, right axis) as a function of salt concentration $c_{ \pm}$as a function of connectivity number $n_{K}$. $a_{ \pm}=0.2 \mathrm{~nm}, a_{P}=0.2 \mathrm{~nm}$, and $d_{P}=0.5 \mathrm{~nm}$. Increasing the connectivity number of the polymer charge $a_{P}$ drastically enhances coacervation. (b) Phase boundaries (solid lines, left axis) and ion partitioning $\lambda$ (dashed lines, right axis) as a function of salt concentration $c_{ \pm}$ as a function of charge spacing $d_{P} / a_{P} . a_{ \pm}=0.2 \mathrm{~nm}, a_{P}=0.2 \mathrm{~nm}$, and $n_{K}=6$. Decreasing the charge spacing $d_{P}$ drastically enhances coacervation.

We likewise can change the distance between connected charges by adjusting $d_{P}$. This corresponds to increasing the spacing of charges along the chain, from being essentially adjacent $\left(d_{P}=0.4 \mathrm{~nm}\right)$ to very distant $\left(d_{P}=2.0 \mathrm{~nm}\right)$. We plot this in Figure $8 \mathrm{~b}$, which demonstrates the variation of $d_{P}$ with constant $a_{P}=0.2 \mathrm{~nm}, a_{ \pm}=0.2 \mathrm{~nm}$, and $n_{K}=6$. This leads to similar behaviors as increasing or decreasing the value of $n_{K}$. As $d_{P}$ increases the region of coacervate formation decreases significantly. This limit of $d_{P} \rightarrow \infty$ is consistent with the limit of $n_{K} \rightarrow 1$, and leads to a disappearance of correlations due to connectivity. Likewise, decreasing the value of $d_{P}$ results in enhanced coacervation. These two effects combine to promote the cooperative interaction of oppositely-charged polyions; there is less entropic penalty to have clusters of charge due to the connectivity between the charges enforcing the local presence of the ions upon initial charge interaction. This leads to significant 
net attraction between the polyions, and subsequently to coacervation.

We emphasize that connectivity correlation is not explicitly included in the VoornOverbeek theory, but is central to counterion release ideas. ${ }^{7,8}$ This theory retains both of these limits, and demonstrates that connectivity correlations play a large role in the formation of a coacervate system. While, in principle this should lead to enhanced coacervation beyond the range of Voorn-Overbeek predictions, ${ }^{7,34}$ this is not observed in our current theory. ${ }^{34}$ To explain this, we demonstrate that excluded volume also plays a key role in determining coacervate thermodynamics, and does so in a fashion that has the opposing effect of suppressing coacervate formation.

\section{Excluded Volume}

Coacervate phase behavior in this model is strongly dependent on the size of the charged group along the polyions. The excluded volume can be substantial in these systems; $1 M$ of a salt with a hydrated radius of $3 \AA$ will exclude $\approx 7 \%$ of the total volume. Here we systematically determine the nature of the bead size in the PRISM model on the thermodynamics of the system. For the polymer, this involves adjusting the size of the charged groups $a_{P}$. These polyion charges are correlated beads, and the meaning of this with relation to the excluded volume inherent to the polymer portion (i.e. the Flory-Huggins aspect) of the model is unclear. Importantly, we note that both the natural log contribution to the chemical potential as well as even the $\chi$-parameter incorporate the finite size of the species involved; the latter through a Mayer integral that naturally incorporates finite size information. ${ }^{56}$ Despite this imprecision, we can still change the size of these charges to change the excluded volume effect in the scope of the LS portion of the calculation, and do so using $a_{ \pm}=0.2 \mathrm{~nm}, d_{P}=0.5 \mathrm{~nm}$, and $n_{K}=6$. We subsequently adjust $a_{P}=0.14-0.2 \mathrm{~nm}$ (we consider smaller sizes to be most relevant due to the inclusion of finite sizes in other aspects of the calculation). Figure 9 a demonstrates the strong effect that changing the size of the charges can have on the scope of the coexistence region. At small values of $a_{P} \rightarrow 0.1 \mathrm{~nm}$, we 
observe a systematic enhancement of coacervation that is profoundly larger than the effects of changing the salt ion size. We attribute this marked change in coacervation to two effects; upon decreasing the size of $a_{P}$ the contact energy $U_{C}$ of two adjacent and oppositely-charged polyions is much larger $U_{C} \sim 1 /\left(2 a_{P}\right)$ increasing the driving force for coacervation. Likewise, the excluded volume of the polyions becomes much smaller; this decreases the suppression of coacervation due to the lower configurational entropy that occurs due to the finite size of these species.
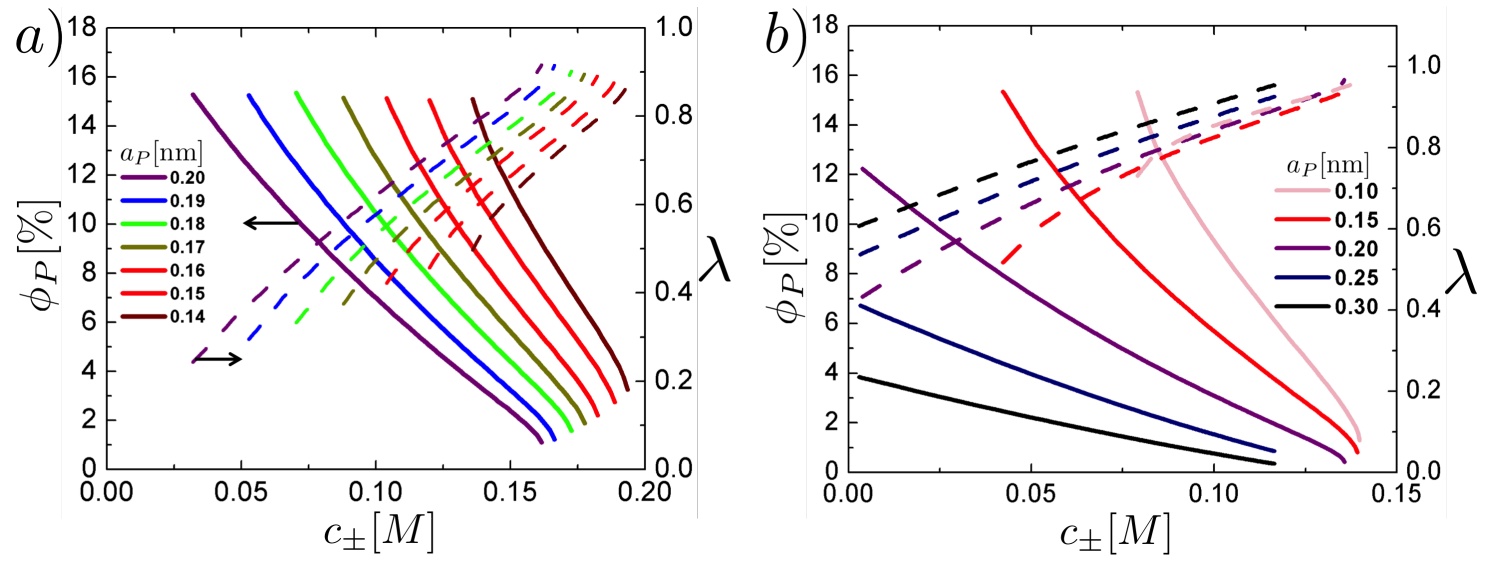

Figure 9: (a) Phase boundaries (solid lines, left axis) and ion partitioning $\lambda$ (dashed lines, right axis) as a function of salt concentration $c_{ \pm}$as a function of polymer charge size. $a_{ \pm}=$ $0.2 \mathrm{~nm}, n_{K}=6$, and $d_{P}=0.5 \mathrm{~nm}$. Decreasing the size of the polymer charge $a_{P}$ drastically enhances coacervation. (b) Phase boundaries (solid lines, left axis) and ion partitioning $\lambda$ (dashed lines, right axis) as a function of salt concentration $c_{ \pm}$as a function of polymer charge size. $a_{ \pm}=0.2 \mathrm{~nm}, n_{K}=6$, and $d_{P}=0.5 \mathrm{~nm}$. In contrast to Figure $9 \mathrm{a}$, the valency $z_{P}$ is changed such that $U_{C} \sim z_{P}^{2} /\left(2 a_{P}\right)$ is constant. $z_{P}=1.0$ for $a_{P}=0.3 \mathrm{~nm}$, however $z_{P}<1.0$ for $a_{P}<0.3 \mathrm{~nm}$. Decreasing the size of the polymer charge $a_{P}$ still drastically enhances coacervation.

To test the extent to which the finite size effects contribute to the observed phase behavior, we vary the system in a similar fashion (with $a_{ \pm}=0.2 \mathrm{~nm}, d_{P}=0.6 \mathrm{~nm}$, and $n_{K}=6$ ). We once more adjust the value of $a_{P}$, however the valency on the polymer charges $z_{P}$ is also varied such that $U_{C} \sim z_{P}^{2} /\left(2 a_{P}\right)$ is constant. We use $z_{P}=1$ for $a_{P}=0.3 \mathrm{~nm}$, and vary $z_{P}$ accordingly with changes in $a_{P}$ (for example, $z_{P}=0.707$ for $a_{P}=0.15$ ). This maintains a constant Coulombic interaction between directly-adjacent polyions, while varying the extent 
of the excluded volume interactions. This is observed in Figure 9b, which demonstrates that there is a strong suppression of coacervation at large values of $a_{P}$. Indeed, finite volume of polyions has a profound effect on coacervation even when controlling for Coulombic interaction strength. Importantly, we note that even in the limit of $\phi_{P} \rightarrow 0$ there is a strong difference in the extent of coacervation. This is apparent due to the non-zero presence of sat ions $c_{ \pm} \gg 0$, which also feel the excluded volume interactions. As $\phi_{P}$ grows, so does the disparity; this trend is due to the localization of the charges due to the charge connectivity, since a high amount of correlation among connected charges clusters the interacting polyions. This effective increase in the local concentration of the polyions leads to a pronounced change in coacervation due to excluded volume effects that surpasses the already-high concentration of species in solution.

To visualize the extent of this effect, we plot the curve $\mu_{\text {exc }}$ versus $\log \left(\phi_{P}\right)$ for the polymer at constant $c_{ \pm}$at both high and low values of $a_{P}$ (Figure 10). In this situation, only $a_{P}$ is varied while other values $\left(a_{ \pm}=0.2 \mathrm{~nm}, n_{K}=6, c_{ \pm}=0.15\right)$ are kept constant as in Figure 9a. At low values of $\log \left(\phi_{P}\right)$, where excluded volume effects are not prevalent, the initial decreasing slope in excess chemical potential (such that the polymer strongly desires to be in a more dense phase) is slightly stronger when $a_{P}$ is smaller due to an increase in the magnitude of the contact energy. Conversely, the eventual increase in excess chemical potential (where the polymer starts to gain less free energy upon being incorporated into the coacervate phase) due to excluded volume effects occurs later for lower values of $a_{P}$. These two effects combine to promote an enhanced degree of coacervation at lower values of $a_{P}$. We emphasize that the differences due to excluded volume appears to be of much larger magnitude than the differences due to enhanced Coulombic attraction. It may be possible to experimentally probe this effect. We note that excluded volume is already present in the mean-field Flory-Huggins (and related) theories. ${ }^{55}$ However, these effects will be magnified by the increased correlation of the volume-excluding species. This may be altered by changing the relative location of bulky groups along the polymer backbone. If the bulky groups are 


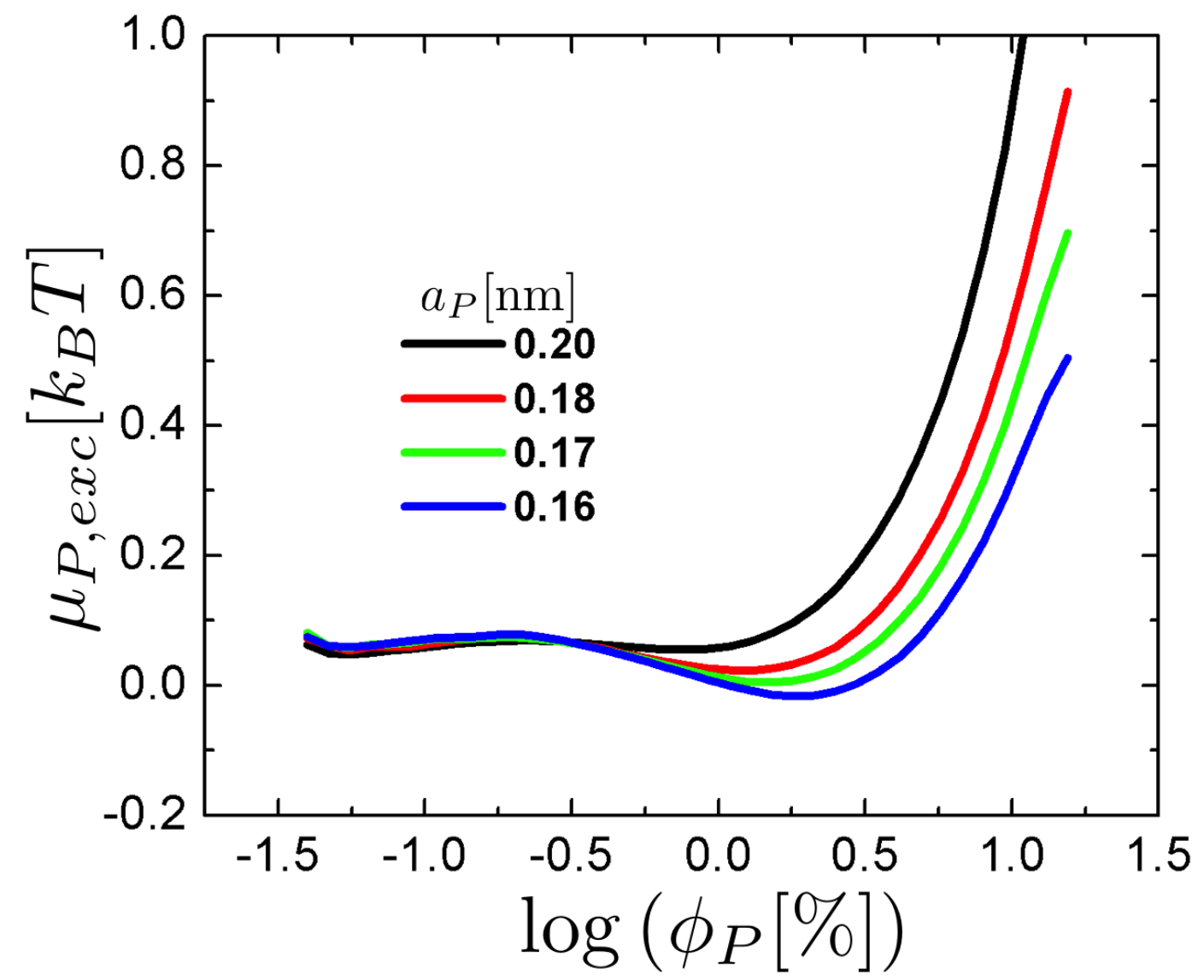

Figure 10: Excess chemical potential of a polyion monomer $\mu_{P, e x c}$ as a function of the log of the polymer concentration $\phi_{P}$. The initial decrease in the chemical potential, which enhances coacervation, is stronger when $a_{P}$ is smaller due to higher electrostatic attraction between the two oppositely-charge polyion species. At high values of $\phi_{P}$, the eventual increase in the chemical potential due to excluded volume effects is weaker with smaller $a_{P}$. These two effects lead to the strong enhancement of coacervation in low- $a_{P}$ systems. 
near charged monomers that are highly correlated, the excluded volume effects would be stronger than if the same bulky groups are far from charged monomers.

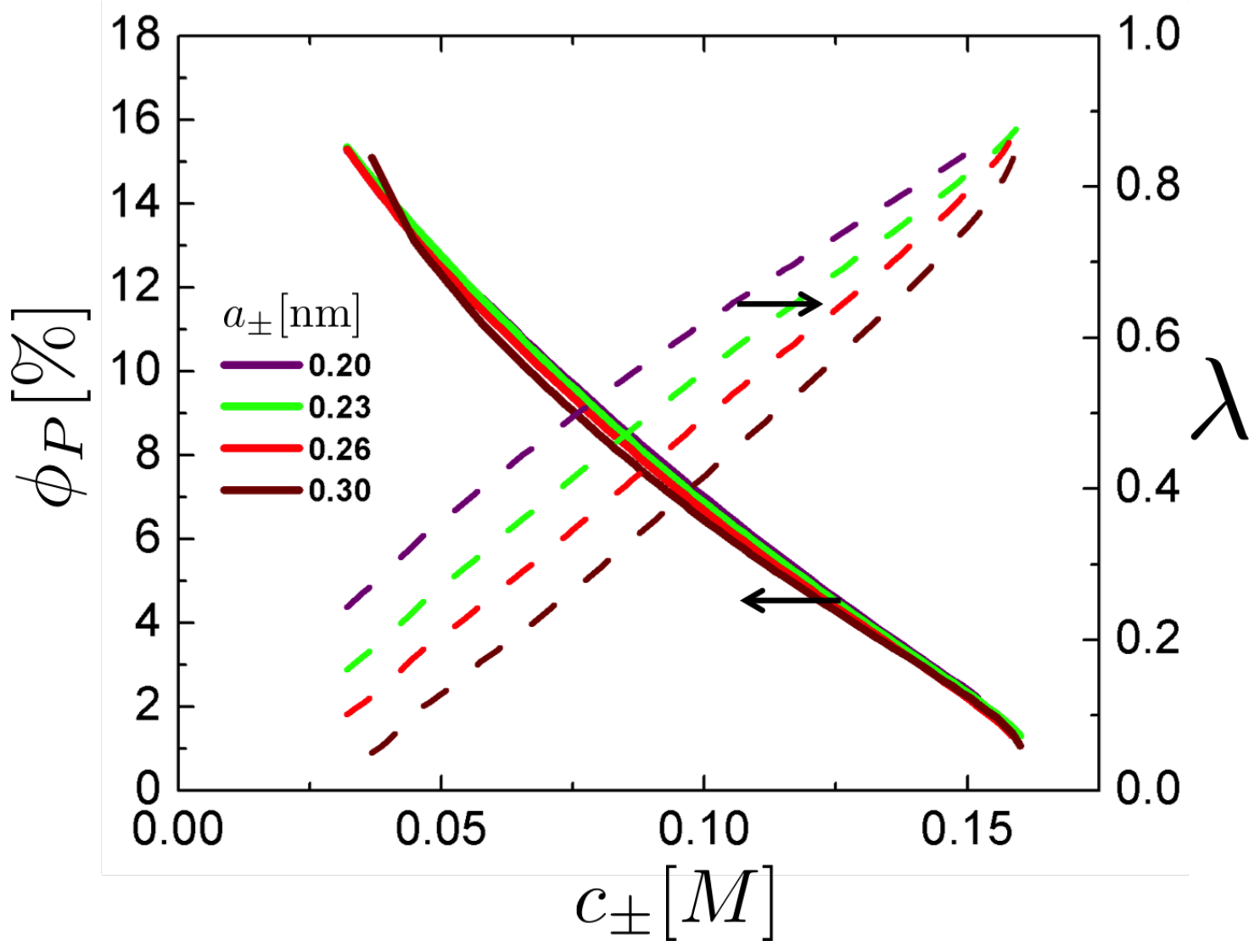

Figure 11: Phase boundaries (solid lines, left axis) and ion partitioning $\lambda$ (dashed lines, right axis) as a function of salt concentration $c_{ \pm}$as a function of salt ion charge size. $a_{P}=0.2 \mathrm{~nm}$, $n_{K}=6$, and $d_{P}=0.5 \mathrm{~nm}$. The value of $\lambda$ strongly decreases as the size of the salt ions is increased, since each ion takes up more of the unoccupied volume in the polymer rich phase. This has very little bearing on the location of the coacervation binodal, which is essentially unchanged. This is attributed to the ability of ions to equilibrate across the phase boundary, such that the increased excluded volume effects due to increased charge size can be mitigated by adjusting the concentration of salt ions in the polymer rich phase.

We can alternatively consider changes in ion size on the thermodynamics of the coacervate phase. This is inherently different than altering the behavior of charge size among the polymeric component, both because there is not a connectivity constraint among the ion charges and also because they are free to equilibrate among the phases. We demonstrate in Figure 11 the trend of charge size for constant values of $a_{P}=0.1 \mathrm{~nm}, d_{P}=0.5 \mathrm{~nm}$, and 
$n_{K}=6$. Here, the size of the ion has little effect on the coacervation behavior. This is due to the equilibration of the salt ions between the two phases. If the salt ions are larger, then less charges can occupy the polymer-rich phase but otherwise the coacervation thermodynamics remain essentially unchanged. In essence, a given polymer chain 'sees' the same salt ion environment in both the polymer rich and poor phases, but in the polymer rich phase it is interacting with the salt ions less due to the presence of other polymer chains. The finite size of the salt ions does dictate their distribution between the two phases. Traditional Voorn-Overbeek theory predicts an abundance of ions in the polymer rich phase rather than the polymer poor phase; this is understandable, since the theory considers no reason not to increase the charge density of the charge-rich phase (after all, the charges are the same for the polymer and the ions!). For our theory (and other theories that include hard sphere or excluded volume interactions) ${ }^{71,72}$ the opposite prediction holds - there must be fewer ions in the polymer rich phase than the polymer poor phase. This is likewise straightforward, since there is not the same amount of unoccupied volume in the polymer rich phase. We demonstrate this in Figures 9a and b. This prediction of fewer salt ions in the coacervate phase represents one of the major departures of our theory from Voorn-Overbeek and related theories.

\section{Conclusion}

We have demonstrated using PRISM-based methods a new way of articulating charge structure in the context of coacervate systems. While we do not make any claims to quantitative matching with experiments, we provide a few trends which appear to provide physically intuitive reasons for the paradoxical state of the theory in complex coacervates. Namely, most theories are related to the Voorn-Overbeek theory, which tends to match extremely well to

experiments. ${ }^{7-9,34}$ We note that these matchings still require a choice for $\chi$ as well as for the strength of the Debye-Hückel term $(\Gamma)$, both of which are used as fitting parameters. Even 
theories that provide substantial improvements on the Voorn-Overbeek theory via impressive field theoretical methods ultimately neglect most of these effects due to their manifestation at truly atomistic levels of correlation that are well below the grid spacing used in these theories. ${ }^{66,67}$ We illustrate that the omission of this length scale results in a cancellation of errors that is primarily due to the neglect of both correlated excluded volume of the polymer chains as well as the similarly-correlated connectivity among the charged polyions. This is demonstrated by the systematically varying both the size and connectivity parameters of our model. Perhaps the most striking difference is the drastically-different distribution of salt ions in this theory. We predict a dearth of charges in the polyion-rich phase, while Voorn-Overbeek predicts the opposite behavior. ${ }^{7,8}$

Our work allows an initial understanding of the ways in which different size and connectivity of charges in coacervates may change equilibrium thermodynamics. We find a number of differences between our results and behaviors predicted in alternative theories; namely, we can directly address the differences due to charge structure that reveal effects due to connectivity and finite charge size. In the appropriate limit, the LS theory reproduces Voorn-Overbeek theory, including the abundance of salt in the polymer-rich phase. Nevertheless, most reasonable parameters do not demonstrate this behavior; rather, salt ions are expelled from the polymer-rich phase due primarily to the excluded volume of the polymer itself. The coacervation is instead driven by the cooperative interactions between the oppositely-charged polyions. We observe strong effects due to polyion size differences, which strongly suppress coacervation via excluded volume.

This rich array of behaviors may be observed in coacervate systems, and is analogous to charged interactions in biological systems that have specific local order driven by connected charges and precise molecular features. ${ }^{44,94}$ Importantly, connectivity behaviors can drastically change the extent of coacervate formation, which is something that we hope to continue to explore in future works. We consider this to be a promising new way to tune self-assembly, with charge connectivity serving as a handle to directly manipulate phase be- 
havior via atomistic-level design. Modulating charge frequency along a polymer backbone, for example, may be used to 'pattern' polyions so that they self-assemble based on how charges are arranged rather than traditional $\chi$-based assembly. The similarity to chargebased biological interactions reveal new opportunities to use this new richness of behavior to emulate biological systems, and varying both charge connectivity and dispersive interactions orthogonally may permit the realization of soft materials with highly specialized interactions and functionality.

\section{Acknowledgement}

The authors thank Matthew Tirrell, Monica Olvera de la Cruz, and Jos Zwanikken for intellectual discussions regarding this work, and Charles Schroeder for his input on the manuscript.

\section{References}

(1) van der Gucht, J.; Spruijt, E.; Lemmers, M.; Cohen Stuart, M.A. J. Coll. Interface Sci. 2011, 361, 407-422.

(2) Stewart, R.J.; Wang, C.S.; Shao, H. Adv. Coll. Interface Sci. 2011, 167, 85-93.

(3) Pergushov, D.V.; Muller, A.H.E.; Schacher, F.H. Chem. Soc. Rev. 2012, 41, 6888-6901.

(4) Wang, Q.; Schlenoff, J.B. Macromolecules, 2014, 47, 3108-3116.

(5) Schaaf, P.; Schlenoff, J.B. Adv. Mater. 2015, 27, 2420-2432.

(6) Shamoun, R.F.; Reish, A.; Schlenoff, J.B. Adv. Funct. Mater. 2012, 22, 1923-1931.

(7) Michaeli, I.; Overbeek, J. Th. G.; Voorn, M.J. J. Poly. Sci. 1957, 23, 443-449.

(8) Overbeek, J. Th. G.; Voorn, M.J. J. Cell. Comp. Phys. 1957, 49, 7-26.

(9) Veis, A.; Aranyi, C. J. Phys. Chem. 1960, 64, 1203-1210. 
(10) Tainaka, K.-I. Biopolymers, 1980, 19, 1289-1298.

(11) Tolstoguzov, V.B. Food Hydrocolloids, 1995, 9, 317-332.

(12) Weinbrek, F.; de Vries, R.; Schrooyen, P.; de Kruif, C.G. Biomacromolecules, 2003, 4, 293-303.

(13) de Kruif, C.G.; Weinbreck, F.; de Vries, R. Curr. Opin. Coll. Interface Sci. 2004, 9, 340-349.

(14) Biesheuvel, P.M.; Cohen Stuart, M.A. Langmuir, 2004, 20, 2785-2791.

(15) Turgeon, S.L.; Schmitt, C.; Sanchez, C. Curr. Opin. Coll. Interface Sci. 2007, 12, 166-178.

(16) Schmitt, C.; Turgeon, S.L. Adv. Coll. Interface Sci. 2011, 167, 63-70.

(17) Anema, S.G.; de Kruif, C.G.K. Soft Matter, 2012, 8, 4471-4478.

(18) Matalanis, A.; Jones, O.G.; McClements, D.J. Food Hydrocolloids, 2011, 25, 1865-1880.

(19) Pittella, F.; Kataoka, K. "Polymeric Micelles for siDNA Delivery." Advance in Delivery Science and Technology (Springer, Berlin, 2012).

(20) Harada, A.; Kataoka, K. Macromolecules, 1995, 28, 5294-5299.

(21) Harada, A.; Kataoka, K. Science, 1999, 283, 65-67.

(22) Cohen Stuart, M.A.; Besseling, N.A.M.; Fokkink, R.G. Langmuir, 1998, 14, 6846-6849.

(23) Krogstad, D.V.; Lynd, N.A.; Choi, S.-H.; Spruell, J.M.; Hawker, C.J.; Kramer, E.J.; Tirrell, M.V. Macromolecules, 2013, 46, 1512-1518.

(24) Krogstad, D.V.; Choi, S.-H.; Lynd, N.A.; Audus, D.J.; Perry, S.L.; Gopez, J.D.; Hawker, C.J.; Kramer, E.J.; Tirrell, M.V. J. Phys. Chem. B 2014, 118, 13011-13018. 
(25) Hunt, J.N.; Feldman, K.E.; Lynd, N.A.; Deek, J.; Campos, L.M.; Spruell, J.M.; Hernandez, B.M.; Kramer, E.J. Adv. Mater. 2011, 23, 2327-2331.

(26) Fan, Y.; Tang, S.; Thomas, E.L.; Olsen, B.D. ACS Nano, 2014, 8, 11467-11473.

(27) Hammond, P.T. Adv. Mater. 2004, 16, 1271-1293.

(28) Lutkenhaus, J.L.; Hrabak, K.D.; McEnnis, K.; Hammond, P.T. J. Am. Chem. Soc. 2005, 127, 17228-17234.

(29) Vidyasagar, A.; Sung, C.; Gamble, R.; Lutkenhaus, J.L. ACS Nano, 2012, 6, 61746184.

(30) Sukhishvili, S.A.; Kharlampieva, E.; Izumrudov, V. Macromolecules, 2006, 39, 88738881.

(31) Lim, S.; Moon, D.; Kim, H.J.; Seo, J.H.; Kang, I.S.; Cha, H.J. Langmuir, 2014, 30, 1108-1115.

(32) Hwang, D.S.; Zeng, H.; Srivastava, A.; Krogstad, D.V.; Tirrell, M.; Israelachvili, J.N.; Waite, J.H. Soft Matter, 2010, 6, 3232-3236.

(33) Wei, W.; Tan, Y.; Martinez Rodriguez, N.R.; Yu, J.; Israelachvili, J.N.; Waite, J.H. Acta Biomater. 2014, 10, 1663-1670.

(34) Spruijt, E.; Westphal, A.H.; Borst, J.W.; Cohen Stuart, M.A.; van der Gucht, J. Macromolecules, 2010, 43, 6476-6484.

(35) Priftis, D.; Tirrell, M. Soft Matter, 2012, 8, 9396-9405.

(36) Priftis, D.; Laugel, N.; Tirrell, M. Langmuir, 2012, 28, 15947-15957.

(37) Priftis, D.; Farina, R.; Tirrell, M. Langmuir, 2012, 28, 8721-8729. 
(38) Priftis, D.; Xia, X.; Margossian, K.O.; Perry, S.L.; Leon, L.; Qin, J.; de Pablo, J.J.; Tirrell, M. Macromolecules, 2014, 47, 3076-3085.

(39) Hwang, D.S.; Waite, J.H.; Tirrell, M. Biomaterials, 2010, 31, 1080-1084.

(40) Black, K.A.; Priftis, D.; Perry, S.L.; Yip, J.; Byun, W.Y.; Tirrell, M. ACS Macro Lett. 2014, 3, 1088-1091.

(41) Chu, H.; Gao, J.; Chen, C.W.; Huard, J.; Wang, Y. Proc. Natl. Acad. Sci. USA 2011, 108, 13444-13449.

(42) Kizilay, E.; Kayitmazer, A.B.; Dubin, P.L. Adv. Colloid Interface Sci. 2011, 167, 24-37.

(43) Kayitmazer, A.B.; Strand, S.P.; Tribet, C.; Jaeger, W.; Dubin, P.L. Biomacromolecules, 2007, 8, 3568-3577.

(44) Kayitmazer, A.B.; Seeman, D.; Minsky, B.B.; Dubin, P.L.; Xu, Y. Soft Matter, 2013, 9, 2553-2583.

(45) Cooper, C.L.; Dubin, P.L.; Kayitmazer, A.B.; Turksen, S. Curr. Opin. Colloid. Interface Sci. 2005, 10, 52-78.

(46) Wang, Y.; Kimura, K.; Huang, Q.; Dubin, P.L.; Jaeger, W. Macromolecules, 1999, 32, 7128-7134.

(47) Chollakup, R.; Beck, J.B.; Dirnberger, K.; Tirrell, M.; Eisenbach, C.D. Macromolecules, 2013, 46, 2376-2390.

(48) Perry, S.L.; Li, Y.; Priftis, D.; Leon, L.; Tirrell, M. Polymers, 2014, 6, 1756-1772.

(49) Jha, P.K.; Desai, P.S.; Li, J.; Larson, R.G. Polymers, 2014, 6, 1414-1436.

(50) Perry, S.L.; Leon, L.; Hoffmann, K.Q.; Kade, M.J.; Priftis, D.; Black, K.A.; Wong, D.; Klein, R.A.; Pierce III, C.F.; Margossian, K.O.; Whitmer, J.K.; Qin, J.; de Pablo, J.J.; Tirrell, M. Nat. Commun. 2015, 6, 6052. 
(51) Hoda, N.; Larson, R.G. Macromolecules, 2009, 42, 8851-8863.

(52) Ou, Z.; Muthukumar, M. J. Chem. Phys. 2006, 124, 154902.

(53) Jeon, J.; Dobrynin, A.V. Macromolecules, 2005, 38, 5300-5312.

(54) Jeon, J.; Dobrynin, A.V. Phys. Rev. E, 2003, 67, 061803.

(55) Flory, P.J., Principles of Polymer Chemistry Cornell Univ. Press: Ithaca, 1953.

(56) McQuarrie, D.A. Statistical Mechanics University Science Books: Sausalito, 2000.

(57) Qin, J.; Priftis, D.; Farina, R.; Perry, S.L.; Leon, L.; Whitmer, J.; Hoffmann, K.; Tirrell, M.; de Pablo, J.J. ACS Macro Lett. 2014, 3, 565-568.

(58) Netz, R.R.; Orland, H. Eur. Phys. J. E. 2000, 1, 203-214.

(59) Raspaud, E.; Olvera de la Cruz, M.; Sikorav, J.-L.; Livolant, F. Biophys. J. 1998, 74, 381-393.

(60) Olvera de la Cruz, M.; Belloni, L.; Delsanti, M.; Dalbiez, J.P.; Spalla, O.; Drifford, M. J. Chem. Phys. 1995, 103, 5781-5791.

(61) Jha, P.K.; Zwanikken, J.W.; Olvera de la Cruz, M. Soft Matter, 2012, 8, 9519-9522.

(62) Sing, C.E.; Zwanikken, J.W.; Olvera de la Cruz, M. Macromolecules, 2013, 46, 50535065.

(63) Sing, C.E.; Zwanikken, J.W.; Olvera de la Cruz, M. Phys. Rev. Lett. 2013, 111, 168303.

(64) Castelnovo, M.; Joanny, J.F. Eur. Phys. J. E 2001, 6, 377-386.

(65) Potemkin, I.I.; Palyulin, V.V. Phys. Rev. E 2010, 81, 041802.

(66) Lee, J.; Popov, Y.O.; Fredrickson, G.H. J. Chem. Phys. 2008, 128, 224908.

(67) Riggleman, R.A.; Kumar, R.; Fredrickson, G.H. J. Chem. Phys. 2012, 136, 024903. 
(68) Audus, D.J.; Gopez, J.D.; Krogstad, D.V.; Lynd, N.A.; Kramer, E.J.; Hawker, C.J.; Fredrickson, G.H. Soft Matter, 2015, 11, 1214-1225.

(69) Manning, G.S. J. Chem. Phys. 1969, 51, 924-933.

(70) Du, X.; Dubin, P.L.; Hoagland, D.A.; Sun, L. Biomacromolecules, 2014, 15, 726-734.

(71) Kudlay, A.; Ermoshkin, A.V.; Olvera de la Cruz, M. Macromolecules, 2004, 37, 92319241.

(72) Kudlay, A.; Olvera de la Cruz, M. J. Chem. Phys. 2004, 120, 404-412.

(73) Burgess, D.J. J. Colloid Interface Sci. 1990, 140, 227-238.

(74) Hansen, J.-P.; McDonald, I.R. Theory of Simple Liquids Elsevier: Boston, 2006.

(75) Yethiraj, A.; Shew, C.-Y. Phys. Rev. Lett. 1996, 77, 3937-3940.

(76) Shew, C.-Y.; Yethiraj, A. J. Chem. Phys. 1999, 110, 11599.

(77) Zherenkova, L.; Khalatur, P.; Yoshikawa, K. Macromol. Theory and Sim. 2003, 12, 339-353.

(78) Hofmann, T.; Winkler, R.G.; Reineker, P. J. Chem. Phys. 2001, 114, 10181.

(79) Zwanikken, J.W.; Jha, P.K.; Olvera de la Cruz, M. J. Chem. Phys. 2011, 135, 064106.

(80) Schweizer, K.S.; Curro, J.G. Phys. Rev. Lett. 1987, 58, 246-249.

(81) Sing, C.E.; Zwanikken, J.W.; Olvera de la Cruz, M. J. Chem. Phys. 2015, 142, 034902.

(82) Sing, C.E.; Zwanikken, J.W.; Olvera de la Cruz, M. Nat. Mat. 2014, 13, 694-698.

(83) Sing, C.E.; Olvera de la Cruz, M. ACS Macro Lett. 2014, 3, 698-702.

(84) Spruijt, E.; Leermakers, F.A.M.; Fokkink, R.; Schweins, R.; van Well, A.A.; Cohen Stuart, M.A.; van der Gucht, J. Macromolecules, 2013, 46, 4596-4605. 
(85) Yethiraj, A.; Schweizer, K.S. J. Chem. Phys. 1992, 97, 1455-1464.

(86) Nair, N.; Jayaraman, A. Macromolecules, 2010, 43, 8251-8263.

(87) Shew, C.-Y.; Yethiraj, A. J. Chem. Phys. 1997, 106, 5706-5719.

(88) Yethiraj, A.; Schweizer, K.S. J. Chem. Phys. 1993, 98, 9080-9093.

(89) Laria, D.; Wu, D.; Chandler, D. J. Chem. Phys. 1991, 95, 4444-4453.

(90) Muthukumar, M.; Hua, J.; Kundagrami, A. J. Chem. Phys. 2010, 132, 084901.

(91) Chaikin, P.M.; Lubensky, T.C. Principles of Condensed Matter Physics Cambridge University Press: Cambridge, 1995.

(92) Nakamura, I.; Balsara, N.P.; Wang, Z.-G. Phys. Rev. Lett. 2011, 107, 198301.

(93) Rubinstein, M.; Colby, R.H. Polymer Physics Oxford University Press: New York, 2003.

(94) Elder, R.M.; Jayaraman, A. J. Phys. Chem. B 2013, 117, 11988-11999. 


\section{Graphical TOC Entry}

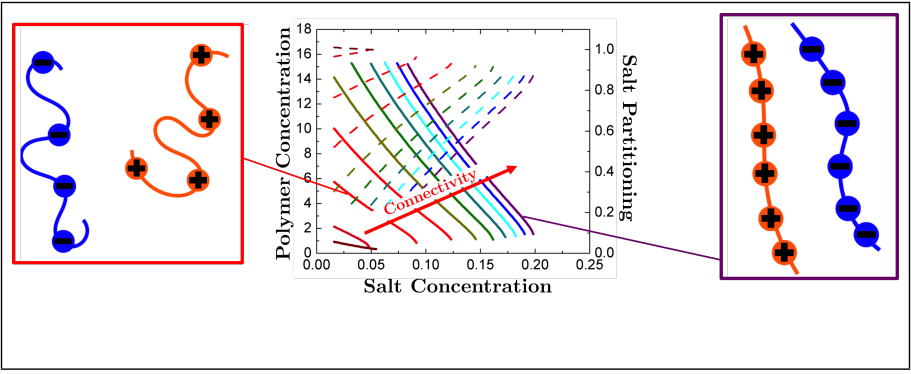

